\title{
GLIA
}

\section{Orthogonal arrays of particle assembly are essential for normal Aquaporin-4 expression level in the brain}

\begin{tabular}{|c|c|}
\hline Journal: & GLIA \\
\hline Manuscript ID & GLIA-00036-2020.R2 \\
\hline Wiley - Manuscript type: & Original Research Article \\
\hline $\begin{array}{r}\text { Date Submitted by the } \\
\text { Author: }\end{array}$ & $\mathrm{n} / \mathrm{a}$ \\
\hline Complete List of Authors: & $\begin{array}{l}\text { De Bellis, Manuela; University of Bari, Department of Bioscience, } \\
\text { Biotechnologies and Biopharmaceutic and Center of Excellence in } \\
\text { Comparative Genomics (CEGBA) } \\
\text { Cibelli, Antonio; University of Bari, Department of Bioscience, } \\
\text { Biotechnologies and Biopharmaceutic and Center of Excellence in } \\
\text { Comparative Genomics (CEGBA) } \\
\text { Mola, Maria; University of Bari "Aldo Moro", Department of Bioscience, } \\
\text { Biotechnologies and Biopharmaceutic and Center of Excellence in } \\
\text { Comparative Genomics (CEGBA) } \\
\text { Pisani, Francesco; University of Bari "Aldo Moro", Department of } \\
\text { Bioscience, Biotechnology and Biopharmaceutics } \\
\text { Barile, Barbara; University of Bari "Aldo Moro", Department of } \\
\text { Bioscience, Biotechnology and Biopharmaceutics } \\
\text { Mastrodonato, Maria; University of Bari, Department of Biology } \\
\text { Banitalebi, Shervin; University of Oslo, Department of Molecular } \\
\text { Medicine, Division of Anatomy } \\
\text { Amiry-Mogghaddam, Mahmood ; University of Oslo, Department of } \\
\text { Molecular Medicine, Division of Anatomy } \\
\text { Abbrescia, Pasqua; University of Bari "Aldo Moro", 4School of Medicine, } \\
\text { Department of Basic Medical Sciences, Neuroscience and Sense Organs } \\
\text { Frigeri, Antonio; Medical Sciences, Neuroscience and sense organ } \\
\text { Svelto, Maria; University of Bari "Aldo Moro", Department of Bioscience, } \\
\text { Biotechnology and Biopharmaceutics } \\
\text { Nicchia, Grazia; University of Bari Aldo Moro, Department of Bioscience, } \\
\text { Biotechnologies and Biopharmaceutic and Center of Excellence in } \\
\text { Comparative Genomics (CEGBA) }\end{array}$ \\
\hline Topics: & Astrocytes, Blood-Brain-Barrier, Homeostasis of extracellular space \\
\hline Techniques: & $\begin{array}{l}\text { Histological techniques, Immunocytochemistry, Transgenic animals, } \\
\text { Molecular Neuroscience }\end{array}$ \\
\hline Key Words: & Astrocytes, Aquaporin-4, Orthogonal Arrays of Particles, CRISPR/Cas9 \\
\hline
\end{tabular}

\section{SCHOLARONE" Manuscripts}


John Wiley \& Sons, Inc. 
Running Title: OAP-null mouse model

\section{Orthogonal arrays of particle assembly are essential for normal}

\section{Aquaporin-4 expression level in the brain}

Manuela De Bellis ${ }^{1, *}$, Antonio Cibelli, ${ }^{1, *}$, Maria Grazia Mola ${ }^{1, *}$,Francesco Pisani ${ }^{1}$, Barbara Barile $^{1}$, Maria Mastrodonato ${ }^{2}$, Shervin Banitalebi ${ }^{3}$, Mahmood Amiry-Mogghaddam $^{3}$, Pasqua $^{2}$ Abbrescia $^{4}$, Antonio Frigeri ${ }^{4}$, Maria Svelto ${ }^{1}$, Grazia Paola Nicchia ${ }^{1, \#}$

${ }^{1}$ Department of Biosciences, Biotechnologies and Biopharmaceutics, University of Bari Aldo Moro, Via Orabona 4, 70125 Bari, Italy.

${ }^{2}$ Department of Biology, University of Bari Aldo Moro, Via Orabona 4, 70125 Bari, Italy.

${ }^{3}$ Department of Molecular Medicine, Division of Anatomy, University of Oslo, P.O Box 1105 Blindern 0317 Oslo, Norway.

${ }^{4}$ School of Medicine, Department of Basic Medical Sciences, Neuroscience and Sense Organs, University of Bari Aldo Moro, Policlinico, Piazza G. Cesare 11, 70124Bari, Italy.

*Manuela De Bellis, Antonio Cibelli and Maria Grazia Mola should be considered joint first author

\#Grazia Paola Nicchia is the corresponding author (email: graziapaola.nicchia@uniba.it)

Acknowledgments This work was supported by a grant from the Italian Ministry of the University and Research (FIRB-Futuro in Ricerca RBFR12SJA8) to GPN. The authors would like to thank Richard Lusardi for his expert assistance in revising the English of the article and Gaetano Devito for excellent technical assistance.

Conflict of interest statement: The authors do not have any potential sources of conflict of interest.

Data Availability Statement: The authors confirm that the data supporting the findings of this study are available within the article.

\section{Word count}

Manuscript 10718; Abstract 238; Introduction 969; Materials and Methods 2261; Results 1637;

Discussion 2011; References 1864

\section{MAIN POINTS}

1) Orthogonal arrays of particle (OAP) assembly is essential for normal Aquaporin-4 expression level in the brain. 2) Most brain AQP4 is organized into OAPs under physiological conditions. 


\begin{abstract}
Astrocyte endfeet are endowed with Aquaporin-4 (AQP4)-based assemblies called Orthogonal Arrays of Particles (OAPs) whose function is still unclear. To investigate the function of OAPs and of AQP4 tetramers, we have generated a novel "OAP-null" mouse model selectively lacking the OAP forming M23-AQP4 isoform. We demonstrated that AQP4 transcript levels were not reduced by using qPCR. BN/SDS-PAGE and Western blot performed on OAP-null brain and primary astrocyte cultures showed the complete depletion of AQP4 assemblies, the selective expression of M1-AQP4-based tetramers and a substantial reduction in AQP4 total expression level. Fluorescence quenching and super-resolution microscopy experiments showed that AQP4 tetramers were functionally expressed in astrocyte plasma membrane and their dimensions were reduced compared to wild-type assemblies. Finally, as shown by light and electron microscopy, OAP depletion resulted in a massive reduction in AQP4 expression and a loss of perivascular AQP4 staining at astrocyte endfeet, with only sparse labelling throughout the brain areas analysed. Our study relies on the unique property of AQP4 to form OAPs, using a novel OAP-null mouse model for the first time, to show that 1) AQP4 assembly is essential for normal AQP4 expression level in the brain and 2) most of AQP4 is organized into OAPs under physiological conditions. Therefore, AQP4 tetramers cannot be used by astrocytes as an alternative to OAPs without affecting AQP4 expression levels, which is important in the physiological and pathological conditions in which OAP aggregation/disaggregation dynamics have been implicated.
\end{abstract}

\title{
KEYWORDS
}

Astrocytes, Aquaporin-4, Orthogonal Arrays of Particles, Assembly, Tetramers, CRISPR/Cas9, Brain 


\section{INTRODUCTION}

One of the key functions of astrocytes is the control of osmotic and volume physiological parameters guaranteed by the water channel protein Aquaporin-4 (AQP4) (Badaut et al. 2014; Benfenati et al., 2011; Mola et al., 2016). AQP4 is mainly localized at astrocytic endfeet (Nielsen et al., 1997) at the interface between the Central Nervous System (CNS) and liquid compartments (Amiry-Moghaddam et al. 2004b; Hoddevik et al. 2017). In addition, there is a minor "perisynaptic pool" of AQP4 which has been proposed to control the extracellular space volume challenged by neuronal activity (Nagelhus and Ottersen, 2013).

The peculiarity of AQP4 is its plasma membrane organization into large well-ordered assemblies called "square arrays" or "orthogonal array of particles (OAPs)", visible by Freeze Fracture Electron Microscopy (FFEM). Interestingly, OAP alterations have been observed in different pathological conditions such as brain tumors, ischemia and Alzheimer's disease (Neuhaus, 1990; Wilcock et al., 2009; Noell et al., 2012). Moreover, the interest in OAP studies has recently increased since the discovery of their involvement in the pathogenesis of Neuromyelitis Optica (NMO), a CNS autoimmune channelopathy whose autoantibodies are only able to attack their antigen AQP4 if arranged in OAPs (Nicchia et al., 2009a; Crane et al., 2011; Papadopoulos and Verkman, 2012).

The molecular nature of OAPs resides in the distinct characteristic of AQP4 of being expressed as different similar isoforms by two operating mechanisms called leaky scanning (Rossi et al., 2010) and translational read-through (De Bellis et al., 2017; Palazzo et al., 2019), able to produce isoforms with a shorter N-terminus or with a longer C-terminus, respectively, as well as by alternative splicing (Moe et al. 2008). 
M1-AQP4 and M23-AQP4 are more abundant and the very well characterized AQP4 isoforms synthetized from two in-frame translation initiation sites, Met-1 for M1-AQP4 and Met-23 for M23-AQP4 (Furman et al. 2003). Two additional AQP4 isoforms have recently been identified, M1-AQP4ex and M23-AQP4ex, each having an extension at the C-terminus (De Bellis et al., 2017; Palazzo et al., 2019). By alternative splicing, six cDNA AQP4 isoforms, named AQP4a-f, can be expressed in rats (Moe et al. 2008), having a different N-terminus and sharing a common Cterminus. AQP4a and AQP4c correspond to the two classical M1- and M23-AQP4 isoforms, respectively.

The major M23- and M1-AQP4 isoforms are very well characterized (Furman et al., 2003; Smith et al., 2014) for their role in OAP formation. When transfected separately, they show similar water permeability (Neely et al., 1999; Silberstein et al., 2004) but different aggregation properties. In particular, M23-AQP4 forms OAPs while M1-AQP4 exists mainly as individual tetramers (Rash et al., 2004). However, when co-expressed, M1-AQP4 can assemble into OAPs together with M23AQP4 as hetero-tetramers, reducing OAP size and affecting their shape (Neely et al., 1999; Crane et al., 2009; Rossi et al., 2012). Interestingly, OAPs have an M23-AQP4-enriched core surrounded by an M1-AQP4-enriched periphery. It thus seems likely that the hydrophobic intermolecular interactions occurring between the shorter N-terminus of M23-AQP4 provide the adhesive properties stabilizing the OAPs, while the additional N-terminus of M1-AQP4 disturbs these interactions, therefore interrupting the increase in OAP dimension (Rossi et al., 2012). Interestingly, an active role of $\mathrm{AQP} 4 \mathrm{e}$ in the regulation of OAP structural dynamics has been recently demonstrated with AQP4e affecting the cell abundance of OAPs in hypoosmotic conditions (Lisjak et al. 2017). However, AQP4e expression, documented in rat, is not predicted in mouse and human due to the presence of a STOP codon upstream of the M1 start codon (Rossi et al. 2011). Moreover, a role in regulating OAP size has been proposed for AQP4ex isoforms (Palazzo et al. 2019). 
It has been proposed that OAP assembly/disassembly dynamics has an impact on astrocytes in health and disease (Amiry-Moghaddam et al. 2004a; Simone et al. 2019; Smith et al. 2014; Verkman et al. 2012). Although M1-AQP4 and M23-AQP4 are always coexpressed in vivo, studies performed in cell cultures transfected with the two isoforms separately have indicated different functional roles of M1-AQP4 based tetramers and M23-AQP4 based OAPs. M1-AQP4 based tetramers or small M1-AQP4 enriched OAPs are mobile in the plasma membrane and have been proposed to rapidly diffuse to the front edge of migrating astrocytes (Smith et al., 2014). In contrast, large stable M23-AQP4 enriched OAPs, are proposed to support the opposite function of cell adhesion as they are important for AQP4 polarization at glial endfoot microdomains (AmiryMoghaddam et al., 2004; Verkman et al., 2012; Smith et al., 2014). Moreover, AQP4 aggregation has recently been shown to influence plasma membrane dynamics as well as to alter cell proliferation, invasiveness, migration, and apoptotic potential in glioma cells (Simone et al., 2019). This indicates a new perspective on the role of AQP4 in brain tumors, which is not necessarily associated with edema formation but rather with AQP4 tetramer and OAP dynamics and their link with the actin cytoskeleton.

Based on the important role of AQP4 aggregation into OAPs in the physiology and pathophysiology of CNS glial cells, we have here used the CRISPR/Cas-9 technology to generate a new mouse model selectively lacking the OAP forming isoform M23-AQP4 and therefore called OAP-null mouse. Given the concerns about the offtarget genes potentially targeted by the use of short gRNAs, we produced two different strains of mice carrying the same mutation but using two diverse and independent strategies. The unaltered expression of AQP4 mRNA, the functional expression of AQP4 tetramers and the inability to form supramolecular assemblies conducible to OAPs have been validated in the two mice strains. Biochemical combined with functional assays and light, super-resolution and electron microscopy experiments have provided important information on the role of AQP4 tetramers and their aggregation into OAPs in the brain under 
normal conditions. We believe this study provides a better mechanistic understanding of the unique property of AQP4 to aggregate into OAPs using a novel OAP-null mouse model for the first time. 


\section{MATERIALS AND METHODS}

Ethics statement. In this study no experiments were performed on live animals. Experiments were performed according to the European directive on animal use for research and the Italian law on animal care. The protocols were approved by the Italian Ministry of Health (Protocol no. 710/2017PR and 571/2018-PR). All experiments were designed to minimize the number of animals used and animal suffering. Mice were maintained under a 12-h dark to light cycle, at constant room temperature (RT) and humidity, with food and water provided ad libitum, and supplied with environmental enrichment materials such as toys and shelters.

Animals. OAP-null mice harboring the M23I point mutation were generated on the C57BL/6J background by Cyagen Biosciences (Santa Clara, CA, USA) using CRISPR/Cas9-based targeting and homology-directed repair. The details of the genome editing approach are described in the Results section. In founder lines, the lack of mutations in off-target genes was verified by PCR amplification and sequencing of selected candidate genes. Intercrossing of heterozygous mice yielded healthy offspring at Mendelian ratios. AQP4 knockout (KO) mice with a CD1 genetic background and age-matched CD1 mice, used as WT mice, were kindly provided by Dr. Hu (Nanjing Medical University, China). Genotyping was performed on tail DNA using standard protocols.

Antibodies. The primary antibodies used were: goat polyclonal anti-AQP4 (C-19, Santa Cruz, Dallas, TX, USA) and rabbit polyclonal anti-AQP4 (H-80, Santa Cruz, Dallas, TX, USA) both diluted 1:400 for immunofluorescence and 1:500 for immunoblot analysis; rabbit anti-AQP4 (A5971, Sigma-Aldrich, Saint Louis, MO, USA) at 1:400 dilution for electron microscopy, and mouse monoclonal anti-GFAP (clone G-A-5, Millipore, Burlington, MA, USA) at 1:500 for 
immunofluorescence. An affinity-purified rabbit polyclonal antibody to a predicted antigen (MSDRAAARRWGKC) within the mouse M1-AQP4 specific sequence was prepared by GenScript (Leiden, Netherlands) and diluted 1:1000 for immunoblot analysis. The following secondary antibodies (all from Santa Cruz, Dallas, TX, USA) were used for Western blot diluted to 1:5000: donkey anti- goat IgG-horse- radish peroxidase (HRP); goat anti-rabbit IgG-HRP. The secondary antibodies used for immunofluorescence were: Alexa Fluor594 donkey anti-mouse, Alexa Fluor488 donkey anti-rabbit and Alexa Fluor488 donkey anti-goat (all from Thermo, Waltham, MA, USA) diluted 1:1000. The secondary antibody used for immunohistochemistry was goat anti-rabbit HRPconjugate diluted 1:100 whereas for immunogold electron microscopy it was goat anti-rabbit antibody coupled to $15 \mathrm{~nm}$ colloidal gold particles (1:20, Abcam, Cambridge, UK).

Astrocyte primary cell culture. Mouse astrocyte primary cultures were prepared from newborn pups as previously described (Nicchia et al., 2000). Cells were cultured in DMEM-Glutamax medium supplemented with $10 \%$ fetal bovine serum (FBS), $100 \mathrm{U} / \mathrm{ml}$ penicillin and $100 \mathrm{mg} / \mathrm{ml}$ streptomycin, and maintained at $37^{\circ} \mathrm{C}$ in a $5 \% \mathrm{CO}_{2}$ incubator.

Brain, cerebellum and astrocyte primary culture protein lysates. Brains were dissolved in seven volumes of $\mathrm{BN}$ buffer (1\% Triton X-100, $12 \mathrm{mM} \mathrm{NaCl,} 500 \mathrm{mM}$ 6-aminohexanoic acid, $20 \mathrm{mM}$ Bis-Tris, pH 7.0, 2 mM EDTA, 10\% glycerol) added with Protease Inhibitor Cocktail (Roche, Indianapolis, IN, USA). A confluent layer of astrocyte primary cultures was washed once with icecold PBS, harvested with a cell scraper, and lysed into seven volumes of BN buffer plus Protease Inhibitor Cocktail (Roche, Indianapolis, IN, USA). After $1 \mathrm{~h}$ incubation on ice the samples were then centrifuged at $21,000 \mathrm{Xg}$ for $30 \mathrm{~min}$ at $4{ }^{\circ} \mathrm{C}$. The supernatants were collected, and the total protein content was calculated using the BCA Protein Assay Kit (Thermo, Waltham, MA, USA). 
Western blotting. Protein lysates were dissolved in Laemmli Sample Buffer (Bio-Rad, Hercules, CA, USA) added with $50 \mathrm{mM}$ dithiothreitol, heated to $37^{\circ} \mathrm{C}$ for $10 \mathrm{~min}$, resolved in a $13 \%$ polyacrylamide gel, and transferred onto polyvinylidenedifluoride (PVDF) membranes (Immobilon PVDF; Millipore, Burlington, MA, USA) for immunoblot analysis. Membranes containing the blotted proteins were blocked with $5 \%$ nonfat dried milk, incubated with primary antibodies for 2 h, washed and incubated with HRP-conjugated secondary antibodies. After washing, reactive proteins were revealed using an enhanced chemiluminescent detection system (ECL-Plus; Pierce Euroclone, Pero, MI, Italy) and visualized on a Chemi-Doc imaging system (Bio-Rad, Hercules, CA, USA). Images were recorded and data analyzed with Image lab software (Bio-Rad, Hercules, CA, USA).

Blue Native (BN)-PAGE and two-dimensional BN/SDS-PAGE. $50 \mu \mathrm{g}$ of protein sample were mixed with 5\% Coomassie Blue-250 and loaded onto a polyacrylamide native gradient gel (3-9\%) (Schaägger et al., 1994). The running buffers were as follows: anode buffer (25 mM imidazole, $\mathrm{pH}$ 7) and blue cathode buffer (50 mMtricine, $7.5 \mathrm{mM}$, imidazole, 0.02\% Coomassie Blue G-250, $\mathrm{pH}$ 7). Electrophoresis was performed at $6 \mathrm{~mA}$ and stopped when the tracking line of the Coomassie Blue G-250 dye had left the edge of the gel. Proteins were blotted onto PVDF membranes (Millipore, Burlington, MA, USA) for immunoblot analysis or alternatively, the lanes were used for the second dimension (2D). For the 2D BN/SDS-PAGE analysis, lanes from the first dimension were cut into individual strips and equilibrated in denaturing buffer $(1 \%$ SDS and $1 \%$ mercaptoethanol) for $1 \mathrm{~h}$ at RT and placed in a 13\% 2D SDS-polyacrylamide gel with the same thickness. At the end of the run, the gel was blotted onto a PVDF membrane (Millipore, Burlington, MA, USA) for Western blot analysis performed as described above. 
Immunofluorescence on tissues sections. For brain sections, the tissue was isolated, fixed in $4 \%$ PFA solution overnight $(\mathrm{O} / \mathrm{N})$ at $4{ }^{\circ} \mathrm{C}$, washed in PBS, immersed in $30 \%$ sucrose solution and frozen at $-80^{\circ} \mathrm{C}$. For immunofluorescence experiments, sections of $10 \mu \mathrm{m}$ thickness were cut on a cryostat (CM 1900; Leica, Wetzlar, Germany) at $-20^{\circ} \mathrm{C}$. After blocking with $0.1 \%$ Gelatin in PBS, sections were incubated with primary antibodies $\mathrm{O} / \mathrm{N}$ at $4{ }^{\circ} \mathrm{C}$ or for $1 \mathrm{~h}$ at $\mathrm{RT}$ in blocking solution. After 30 min of washing, sections were incubated with secondary antibodies for $1 \mathrm{~h}$, washed again for 15 min in PBS and mounted with Mowiol or in PBS-glycerol (1:1) pH 8.0, containing 1\% npropylgallate. Immunostained tissues were observed with a Leica DM6000B microscope equipped with HC PL Fluotar 10x and 40x objectives. In all experiments, no-primary and no-secondary antibody controls were run in parallel. No specific staining was observed in these controls.

Stimulated emission depletion (STED) microscopy and data analysis. Immunofluorescence for gated STED (gSTED) was performed as reported in the Quick Guide to the STED Sample Preparation (http://www.leica- microsystems.com). Briefly, the cells were washed with PBS, fixed with 2\% PFA (Sigma-Aldrich, Saint Louis, MO, USA) in PBS for $10 \mathrm{~min}$ and permeabilized with $0.3 \%$ Triton X-100 (Sigma-Aldrich, Saint Louis, MO, USA) for $10 \mathrm{~min}$. Cells were saturated with 2\% BSA (Sigma-Aldrich, Saint Louis, MO, USA) in PBS, incubated with primary antibodies in 2 \% BSA in PBS, washed and incubated with secondary Alexa Fluor-conjugated antibodies (Thermo, Waltham, MA, USA) in 2\% BSA in PBS. After three washings with PBS cells were finally mounted with Mowiol. A Leica TCS SP8 STED 33 microscope and Leica LASX software were used for image acquisition and analysis (Leica Microsystems CMS GmbH, Wetzlar, Germany). A Leica HC PL APO 1003/1.40 Oil STED White objective and Type F Immersion liquid with a refractive index of 1.5 were used. Excitation of the Alexa Fluor488 dye was achieved with a continuous wave at $488 \mathrm{~nm}$ using a diode laser with a maximum light output in the focal plane of 10 
$\mathrm{mW}$ (NKT Photonics supercontinuum laser). Depletion was performed using a fiber laser with a continuous wave of $592 \mathrm{~nm}$ and a maxim light output in the focal plane of $<0.5 \mathrm{~mW}$ (Laser Quantum). Multiple random regions of interest (ROI) $(\sim 20 \times 20 \mu \mathrm{m}$ sized) were taken using extra optical zoom (5-7) and imaged by confocal and gSTED microscopy. A minimum of 4 ROIs was chosen per cell from representative cell images and used for the analysis. STED images were deconvoluted in Huygens Professional Software (Scientific Volume Imaging) by using the Deconvolution Wizard plug-in, specifying a minimum signal-to-noise ratio of 40 and number of iterations of 100. After threshold adjustment, the image processing package Object Analyzer was used to determine the AQP4-aggregate size, expressed as cluster surface $\left(\mathrm{nm}^{2}\right)$, while the density of AQP4-clusters is expressed as the number of aggregates normalized to the cell surface area. 4-5 cells were analyzed for each independent experiment and condition. Three independent experiments were conducted.

Tissue morphology and Immunohistochemistry. Brain samples were fixed in $10 \%$ of neutral buffered formalin, dehydrated using a graded ethanol series and then embedded in paraffin wax. Serial sections $5 \mu \mathrm{m}$ thick were cut. Rehydrated sections were stained using Mallory's trichrome method for distinguishing cellular from extracellular components. Collagen fibers stained an intense blue, cytoplasm stained reddish, and nucleoli stained pinkish. Images were captured in bright light using an Eclipse E600 photomicroscope and a DMX1200 digital camera (Nikon Instruments SpA, Calenzano, Italy). Pictures of OAP-null and WT brain and cerebellum were taken, under different magnifications to obtain the same fields, in order to assess morphological alterations between WT and OAP-null tissue.

The sections were rehydrated in graded ethanol for immunohistochemistry. Endogenous peroxidases were blocked by incubating sections in PBS containing $1 \% \mathrm{H}_{2} \mathrm{O}_{2}$ for 30 min at RT. After several washes in PBS, the sections were treated with the blocking buffer $1 \%$ normal goat 
serum (Sigma-Aldrich, Saint Louis, MO, USA) in PBS, for $30 \mathrm{~min}$ at RT. After several washes in PBS, sections were incubated in blocking buffer $\mathrm{O} / \mathrm{N}$ at $4{ }^{\circ} \mathrm{C}$ with anti-AQP4 antibody (Santa Cruz Biotechnology, Dallas, TX, USA, goat polyclonal IgG). After several rinses in PBS, sections were incubated with the secondary antibody goat anti-rabbit HRP-conjugate for $1 \mathrm{~h}$ at RT. After several washes in PBS, the immunolabeling was visualized by incubation with 3-30-diaminobenzidine$\mathrm{H}_{2} \mathrm{O}_{2}$ medium for $10 \mathrm{~min}$ at RT. Finally, two sections per slide were counterstained with Hematoxylin and then dehydrated, cleared, and mounted with Entellan. Negative controls were performed by omitting the primary antibodies.

Perfusion and tissue processing for electron microscopy. Mice were deeply anesthetized with an intraperitoneal injection of ketamine $(100 \mathrm{mg} / \mathrm{kg}$ body weight $)$ and transcardially perfused with a solution of $4 \%$ formaldehyde and $0.1 \%$ glutaraldehyde in $0.1 \mathrm{M}$ Phosphate Buffer (PB). The brains were dissected out, post-fixed $\mathrm{O} / \mathrm{N}$ in the fixation solution and stored in a 1:10 dilution of the same solution. Tissue blocks of 1 x $1 \mathrm{~mm}$ from parietal cortex and cerebellum were subjected to the postembedding procedure as described previously (Lunde et al 2015). Briefly, the tissue blocks were cryoprotected in a graded glycerol solution $(10 \%, 20 \%$ and $30 \%$ glycerol in $0.1 \mathrm{M} \mathrm{PB})$, rapidly frozen in liquid propane $\left(-170{ }^{\circ} \mathrm{C}\right)$ and subjected to freeze substitution. Methacrylate resin (Lowicryl HM20) was used to embed the specimens and subjected to UV light for polymerization below $0{ }^{\circ} \mathrm{C}$. Ultrathin sections of $80-100 \mathrm{~nm}$ were cut using an ultratome (Reichert Ultracut $\mathrm{S}$, Leica, Wetzlar, Germany) and the sections were placed on 300 mesh grids.

Immunogold electron microscopy. Immunogold labeling was performed as previously described (Lunde et al., 2015; Hoddevik et al., 2017). Briefly, the sections were incubated O/N with primary antibody, followed by incubation with secondary antibody coupled to $15 \mathrm{~nm}$ colloidal gold particles 
for $90 \mathrm{~min}$. The sections were contrasted with $2 \%$ uranyl acetate and $0.3 \%$ lead citrate. The sections were examined and electron micrographs were acquired using a transmission electron microscope (TEM; Tecnai 12, FEI Company, Eindhoven, Netherlands) at $80 \mathrm{kV}$.

Quantitative PCR. RNA extraction was carried out using Trizol Reagent (Invitrogen, Milan, Italy) according to the instruction manual. Total RNA was quantified by Nanodrop (Thermo, Waltham, MA, USA). $1 \mu \mathrm{g}$ of total RNA was retro transcribed by SuperScript III Reverse Transcriptase (Invitrogen, Milan, Italy). AQP4 total expression was measured by the GAPDH normalized DDCt quantification method using the following primers targeting AQP4 exon 1 (AQP4 For: CGGTTCATGGAAACCTCACC; AQP4 Rev: CATGCTGGCTCCAGTATAAT; GAPDH For: TGCACCACCAACTGCTTAGC; GAPDH Rev: GGCATGGACTGTGGTCATGA). Power up Sybr Green (Invitrogen, Milan, Italy) and StepOne (Applied Biosystems, Thermo, Waltham, MA, USA) were used. All reactions were run in triplicate. After statistical analysis, the data from the different experiments were plotted and averaged in the same bar graph.

Water transport measurements. Volume changes were measured using the validated Calcein fluorescence quenching approach (Hamann et al., 2002; Solenov et al. 2004) using the Flex Station3 plate reader (http://www.moleculardevices.com) equipped with an automatic liquid handling module able to analyze real time fluorescence kinetic data in the 96-well format (Fenton et al. 2010) (Mola et al., 2009, 2016;) . Astrocytes were seeded at a density of 20,000 cells per well and loaded with $10 \mu \mathrm{M}$ of Calcein-AM $24 \mathrm{~h}$ after plating at which time they were $80-85 \%$ confluent. Cells were rinsed in isotonic PBS and hypertonicity was applied $15 \mathrm{~s}$ after the beginning of data acquisition by adding an appropriate volume of hypertonic (D-mannitol $0.5 \mathrm{M}$ ) solution in order to obtain $360 \mathrm{mOsm} / \mathrm{L}$ final extracellular osmolarity. The changes in fluorescence intensity were directly proportional to changes in cell volume. The signal decreases upon addition of hypertonic 
solution as a consequence of water efflux and cell shrinkage. Data acquisition was performed using SoftMax Pro software v5.3, and the data were analyzed with Prism (Graph Pad, San Diego, CA, USA) software. The osmotic properties of the glial cell plasma membranes were analyzed by comparing the time constant obtained by fitting the data with an exponential function.

Experimental design and statistical analysis. All data represent replicates from independent experiments whose number is provided in the figure legends. Statistical analysis was performed in RStudio and GraphPad4 software. First, a Shapiro-Wilk normality test was performed on the data, then statistical significance was evaluated using the unpaired Mann-Whitney test for non-normally distributed data, while the unpaired Student's $t$-test or ANOVA with multiple statistical comparisons between groups (Newman-Keuls test) were performed for normally-distributed data. $P$-values are defined as follows: $* P<0.05, * * P<0.01$, and $* * * P<0.001$. 


\section{RESULTS}

\section{OAP-null mouse model generated by CRISPR/Cas9}

The CRISPR/Cas9 genome editing approach was used to introduce the point mutation (ATG $\rightarrow$ ATC) in a C57BL/6 mouse model in order to obtain a knock-in (KI) mouse having a M23I substitution in the Aqp4 locus at the coding sequence of exon 1 (Ma et al., 1997) (Fig1). Given the concerns about off-target genes potentially targeted by the use of short gRNAs, we decided to generate two different strains bringing the same amino acid substitution of methionine 23 (ATG) to isoleucine (ATC), using two gRNAs (gRNA1 and gRNA2) targeting different AQP4 sequences (Fig 1A). As the $\mathrm{G} \rightarrow \mathrm{C}$ mutation matches the last position for gRNA1, gRNA1 was co-injected with an oligo donor containing an additional silent mutation at the PAM sequence (TCC $\rightarrow$ TCA) to prevent the binding and re-cutting of the sequence by the same gRNA1 after homology-directed repair. In contrast, as the $\mathrm{G} \rightarrow \mathrm{C}$ mutation matches approximately in the middle for gRNA2, gRNA2 was coinjected with an oligo donor not containing any mutation at the PAM sequence (Fig 1B). After the heterozygous were obtained for both strains, the desired mutations in Aqp4 gene were confirmed by DNA sequencing (Fig1C), and heterozygous mice were bred to obtain homozygous offspring. The analysis of live offspring showed a genotype distribution that did not differ significantly from the Mendelian ratio, indicating the absence of pre- or perinatal lethality. Moreover, the animal growth was normal and no sign of suffering phenotype was revealed, as for AQP4 KO mice totally lacking the AQP4 gene (Ma et al., 1997; Fan et al., 2005; Haj-Yasein et al., 2011).

M23I mutation does not affect AQP4 mRNA level and impairs OAP assembly in brain and cerebellum of both strains of OAP-null mice 
The validation and characterization of the two strains of OAP-null mice (indicated as OAP-null.1 and OAP-null.2) was initially performed by qPCR, BN-PAGE and 2D BN/SDS-PAGEImmunoblotting (Fig 2). The first analysis was aimed at verifying whether AQP4 mRNA expression levels were unchanged in OAP-null mice despite the point mutation in the AQP4 gene. The analysis of the total level of AQP4 mRNA performed in the brain and cerebellum of OAP-null and WT mice indicated that M23I mutation did not affect AQP4 gene transcription, leaving AQP4 mRNA levels unaltered compared to WT (Fig 2A).

The presence of multiple AQP4 assemblies, ascribable to AQP4-OAPs of different size (AmiryMoghaddam et al., 2004; Nicchia et al., 2004; Crane et al., 2009), was then analyzed in OAP-null.1 and OAP-null.2 by BN-PAGE followed by immunoblotting using the commercially available antibodies against AQP4 C-terminus (AQP4 Ab) (Fig 2B). The results show that while in all WT tissues several separate bands corresponding to OAPs of different sizes were visible as expected, only a single band corresponding to the smallest AQP4 pool (AQP4 tetramers) was detected in both strains of OAP-null mouse tissues. Densitometric analysis selectively performed on AQP4 tetramers demonstrated an up-regulation in OAP-null mice compared to WT quantified at more than three times higher in the brain and more than twice as high in the cerebellum. The composition of the single AQP4 lower molecular weight pool (AQP4 tetramer) in both strains of OAP-null mice was later analyzed by two-dimensional BN/SDS-PAGE (Fig 2C) followed by immunoblotting performed with AQP4 Ab. Membrane proteins were separated on a first dimension performed under native conditions (BN-PAGE) and a second dimension under denaturing conditions (SDS-PAGE). AQP4 immunoblotting showed several distinct spots containing both M1-AQP4 and M23-AQP4 isoforms with variable expression levels in WT mice. M23-AQP4 was predominant in the largest pool with its expression showing a progressive reduction in the smaller pools. Concomitantly, the M1-AQP4/M23-AQP4 ratio (32 kDa/30 kDa) increased progressively, reaching its maximal level in the smallest pool (arrows), as previously reported (Nicchia et al., 2008). Results obtained in both 
OAP-null mouse strains, in line with the BN/PAGE, showed the selective presence of an upregulated smallest pool and the complete depletion of AQP4 assemblies. The smallest pool, likely corresponding to AQP4 tetramer, was made of both M1-AQP4 and M23-AQP4 (32-30 kDa) in WT mice while it was exclusively made of M1-AQP4 $(32 \mathrm{kDa})$ in both strains of OAP-null mice. The separation of M1-AQP4 and M1-AQP4ex could be generally visualized in immunoblot with a good detection efficiency. No detectable AQP4 protein was revealed in parallel by immunoblot analysis of tissues from AQP4 KO mice.

Western blot experiments performed with AQP4 Ab on brain and cerebellum lysates revealed that both strains of OAP-null mice showed the expected selective loss of the $30 \mathrm{KDa}$ band corresponding to the OAP forming isoform M23-AQP4 (Fig 3A). The AQP4 signal at $32 \mathrm{kDa}$, corresponding to the M1-AQP4 isoform also appeared strongly reduced. The ten times higher OAPnull brain and cerebellum protein sample allowed the correct visualization of M1-AQP4 isoform expression together with the expression of the M1-AQP4ex isoform and a protein band slightly smaller than that of the $30 \mathrm{kDa}$, most likely corresponding to the intracellular AQP4f. An unclear separation between the $32 \mathrm{kDa}(\mathrm{M} 1-\mathrm{AQP} 4)$ and the $30 \mathrm{kDa}$ (M23-AQP4) isoforms usually impaired M1-AQP4 quantitative analysis in WT tissues. With the aim of correctly quantifying M1-AQP4 reduction in OAP-null mice, we generated custom-made antibodies against the N-terminus of M1AQP4 (M1Ab) therefore able to recognize the M1-AQP4 specific sequence selectively. Quantitative analysis of the Western blotting experiments performed with M1 Ab showed that the reduction in M1-AQP4 was approximately $85 \%$ in the brain and 90\% in the cerebellum (Fig. 3 A). These results indicate that in both OAP-null mouse strains the deletion of M23-AQP4 also impairs the amount of M1-AQP4, likely to be that devoted to the OAPs under normal conditions (Fig 3B).

\section{M1-AQP4 based tetramers form functional water channels in brain astrocytes of OAP-null mice}


Astrocyte primary cultures from OAP-null mice were used to assess whether M1-AQP4, in the absence of its molecular partner M23-AQP4, could be normally expressed and form tetramers in the plasma membrane, in a condition in which AQP4 polarization is lost (Nicchia et al., 2000; Solenov et al., 2004). OAP-null astrocyte primary cultures were analyzed for AQP4 assemblies, for M1AQP4 and M23-AQP4 expression and then were used to assess the functionality of AQP4 tetramers by water transport assay (Fig 4). BN-PAGE-Immunoblotting experiments (Fig 4A) showed that AQP4 tetramers were approximately three times as upregulated in OAP-null compared with WT astrocytes. Western blot experiments performed with AQP4 Ab confirmed that in astrocytes from OAP-null mice no detectable M23-AQP4 protein was found while those performed with M1Ab revealed that M1-AQP4 isoform expression in OAP-null mice was about 30\% of the expression level observed in WT mice (Fig 4B).

Fluorescence quenching-based assay was used to compare osmotic volume change rate between OAP-null astrocytes and WT (Fig 4C). The kinetics of cell shrinkage in response to hypertonic challenge was slower in OAP-null $(\tau=4.9 \pm 0.03 \mathrm{~s})$ compared to WT astrocytes $(\tau=3.9 \pm 0.4 \mathrm{~s})$ but faster than those acquired in AQP4 KO astrocytes $(\tau=6.2 \pm 0.22 \mathrm{~s})$. The reduced cell volume shrinkage kinetics in OAP-null astrocytes was in line with the absence of the large majority of AQP4. In contrast, no significant difference was found between WT, OAP-null and AQP4 KO astrocytes in terms of maximal amplitude of cell shrinkage during hypertonic stimulus, in line with previous data (Solenov et al., 2004; Mola et al., 2016). Water transport measurements therefore indicate that M1-AQP4 based tetramers are functionally expressed in the plasma membrane of OAP-null astrocyte primary cultures.

\section{M1-AQP4 based tetramers form smaller aggregates in brain astrocytes of OAP-null mice}

Immunofluorescence experiments shown in Fig 5 were performed to investigate differences in 
AQP4 aggregate size between WT and OAP-null astrocytes by the use of gSTED microscopy. The analysis of AQP4 clusters was performed using AQP4 Ab able to recognize all AQP4 isoforms included AQP4 extended isoforms and the intracellular isoforms. By confocal microscopy analysis, WT astrocytes showed the typical AQP4 plasma membrane pattern and no detectable staining was found in astrocytes from AQP4 KO mice. Astrocytes from OAP-null mice showed a generally weaker AQP4 signal than WT astrocytes (Fig 5A). The size and the distribution analysis of AQP4 assemblies at nanoscale level determined by gSTED microscopy showed a different distribution of AQP4 clusters between WT and OAP-null astrocytes (Fig 5 B, C). In particular, significant differences in AQP4-aggregate median size between genotypes were revealed, compatible with the reduction in the size of AQP4 assembly expected for M1-AQP4 tetramers in the absence of M23AQP4. In line with Western blot results, the density of AQP4 aggregates was significantly reduced in OAP-null astrocytes compared with WT (Fig 5 D).

\section{Limited AQP4 tetramer expression in brain and cerebellum astrocytes}

The massive reduction in AQP4 total expression level, although associated with AQP4 tetramer upregulation in OAP-null mice compared with the WT, induced us to perform immunolocalization experiments aimed at looking for potential specific sites for AQP4 tetramer expression in the brain and cerebellum. Meanwhile a morphological analysis of tissues was performed using histology (Fig 6). From both immunofluorescence and immunocytochemistry, AQP4 tetramers were absent at the perivascular endfeet of the brain cortex where the majority of AQP4 is normally expressed, indicating that M23-AQP4 is here required for the perivascular expression of both M23-AQP4 and M1-AQP4. A very faint and diffuse staining was detectable in OAP-null cerebellum by both immunofluorescence and immunocytochemistry. Morphological analysis revealed no histological differences were found between WT and OAP-null brain and cerebellum. 
Ultrastructure analysis was therefore performed to better ascertain potential specific localization for M1-AQP4 based tetramers. Electron microscopic immunogold investigation of the neocortex (Fig 7), and cerebellum (Fig 8) showed only a few residual immunogold particles in the astrocytic endfeet processes abutting the vessels and pial surface in the area analyzed. We also observed a reduction in the AQP4 immunogold labeling in the astrocytic processes in the neuropil. Immunolocalization studies therefore indicate the absence of preferential AQP4 tetramer expression in the area analyzed. 


\section{DISCUSSION}

The extraordinarily high level of expression of AQP4 water channels at the interfaces between CNS and either blood or cerebrospinal fluid is not unexpected given the important fluid movement in and out of the CNS associated with neuronal activity. However, the need for AQP4 to form quite large supramolecular aggregate OAPs in the plasma membrane is a question still unresolved.

In this study, we have generated a novel mouse model selectively expressing AQP4 under its tetrameric form by introducing a point mutation (M23I) able to abolish the translation of the major OAP forming isoform M23-AQP4, leaving unaltered the possibility to produce the major tetramer forming M1-AQP4 isoform. The aim was to obtain information on the role of M23-AQP4 assembly into OAPs and to investigate whether AQP4 tetramers play a role in CNS astrocytes and can be considered as an alternative to AQP4-OAPs.

Before generating the transgenic mouse, the point mutation able to impair the synthesis of M23AQP4 (M23I), while leaving unaltered the synthesis of M1-AQP4, was characterized in HeLa cells. M1-AQP4 carrying the M23I mutation in transfected cells was shown to be expressed at the same level as wild-type M1-AQP4, to be correctly targeted to the plasma membrane and to give rise to functional AQP4 tetramers unable to aggregate into OAPs (Rossi et al. 2010). Results obtained in cell cultures gave us the rationale to generate the OAP-null mouse model using the same genome design approach. Besides the deletion of the major M23-AQP4 isoform, M23I point mutation was predicted to impair the expression of the minor M23-AQP4ex and the intracellular AQP4d isoforms. Consequently, this model was predicted to selectively express the M1-AQP4 isoform together with M1-AQP4ex, and the expected intracellular spliced variants (AQP4b and AQP4f). The two strains of OAP-null mice gave identical results in terms of phenotype, indicating their specificity for the M23I mutation. 
The first information provided by the biochemical analysis of OAP-null brain astrocytes is that in the absence of M23-AQP4, AQP4 tetramer expression increases whereas the total amount of M1AQP4 is significantly reduced with a massive reduction in the total AQP4. Considering that AQP4 transcription was unaltered in OAP-null mice, a reasonable interpretation of these results could be that reduction in the OAPs is the consequence of the missing M23-AQP4, which additionally results in extra "free" M1-AQP4 forming tetramers and that the majority of M1-AQP4 normally devoted to being part of the OAP assembly, is that which is lost in OAP-null mice, as described in Fig 3B. We can therefore speculate that the large majority of M1-AQP4 in the WT CNS is normally aggregated with M23-AQP4 while the amount of AQP4 tetramers is a minor pool of AQP4. Given that the upregulation of AQP4 tetramers in OAP-null mice is approximately twice that of the WT (2X) and also includes the intracellular AQP4 isoforms which do not form OAPs and that the downregulation of M1-AQP4 protein is approximately eight times higher (8X), we can approximately assume that less than $1 / 16(<6 \%)$ of the total of M1-AQP4 protein is devoted to forming tetramers in the WT while the large majority ( $>94 \%$ ) is part of the OAPs, at least in mouse and human. In rat, and possibly other animals, this proportion could be different due to the expression of AQP4e (Lisjak et al., 2017). It has to be highlighted, however, that an indirect role of intracellular AQP4b and AQP4d isoforms in OAP dynamics has been recently indicated (Lisjak et al., 2020). From the point of view of the molecular physiology of AQP4, this suggests that the assembly of AQP4 into OAPs is a key feature characterizing the molecular identity of this protein channel while the amount of AQP4 tetramers is reduced compared to OAPs under normal conditions. These data also indicate that OAP aggregation is fundamental to secure astrocytes a normal level of AQP4 expression in the plasma membrane and therefore AQP4 tetramers cannot be used by astrocytes as an alternative to OAPs without affecting AQP4 expression levels. This is important in the CNS under physiological and pathophysiological conditions in which OAP aggregation/disaggregation dynamics are implicated, such as astrocyte migration and polarization, brain tumors and Neuromyelitis Optica. 
We here show that although affected by the deletion of M23-AQP4, M1-AQP4 based tetramers can be expressed as functional water channels in astrocytes. This was not unexpected given the large number of studies showing that when transfected separately, M1-AQP4 and M23-AQP4 can form functional water channels (Jung et al., 1994; Furman et al., 2003; Jin et al., 2011; Smith et al., 2014) with a comparable water permeability coefficient (Silberstein et al., 2004).

Light and electron microscopic analysis confirmed the importance of OAPs for AQP4 expression, corroborating the indication that transition from OAPs to AQP4 tetramers would not ensure astrocytes the same level of AQP4 expression. The limited possibilities for M1-AQP4 based tetramers to be expressed in the plasma membrane in the absence of their molecular partner M23AQP4 support the model proposed by Smith and Verkman (2015) that M23-AQP4 and OAPs participate in macromolecular complex formation whereas isolated M1-AQP4 tetramers do not interact strongly with other proteins. We suggest that M23-AQP4 is required for proper M1-AQP4 localization within the OAPs by stabilizing M1-AQP4. This proposed "scaffolding role" of M23AQP4 for M1-AQP4 is consistent with the known strong hydrophobic interactions between the two isoforms (Crane et al., 2009). Notably, the protein scaffolding role has a particular importance for the polarized expression of protein assemblies or complexes in perivascular domains. For instance, alpha syntrophin is considered an important molecular scaffold for the correct assembly of several components of the dystrophin associated protein complex in the perivascular astrocyte domain, including AQP4 (Bragg et al., 2006). We can speculate a sort of domino effect in the scaffolding role of alpha syntrophin on M23-AQP4 in turn acting on M1-AQP4.

Two main functional roles have been ascribed to AQP4 aggregation in OAPs in brain physiology also in the light of previous FFEM data reporting the perivascular microdomains to be characterized by the largest OAPs (Furman et al., 2003). The first is to make AQP4 almost immobile, an 
important property to sustain AQP4 polarized expression at glial endfoot microdomains not delimitated by tight junctions. The second is the substantially high water permeability associated with the packed expression of AQP4 water channels at astrocyte endfeet (Yang and Verkman, 1997). The use of primary astrocyte cultures prepared from the OAP-null mice helped us to answer the question as to whether or not the importance of OAP aggregation is directly associated to AQP4 polarized expression (Smith and Verkman, 2015). It has in fact been largely demonstrated that when astrocytes are placed in culture the polarized expression of AQP4 is completely lost (Nicchia et al., 2000; Solenov et al., 2004). The results obtained in astrocyte primary cultures show that the deletion of M23-AQP4 determines a similar trend, as found in brain and cerebellum, with an upregulation of AQP4 tetramers and a significant reduction in M1-AQP4. This indicates that AQP4 expression under its tetrameric form in astrocyte plasma membrane is massively reduced compared to the possibility for AQP4 expression under the OAP form, even in the absence of AQP4 polarized expression. However, based on the higher AQP4 tetramer upregulation and the lower M1-AQP4 downregulation in OAP-null primary cultured astrocytes compared to OAP-null brain and cerebellum, it is likely that the amount of M1-AQP4 devoted to the OAPs is lower in astrocyte cultures (non polarized AQP4 expression) compared with brain and cerebellum astrocytes (AQP4 polarized expression). This indicates that it is likely that an additional need for AQP4 aggregation is associated with AQP4 polarized expression.

Besides the CNS, AQP4 is expressed in various organs such as the skeletal muscle (Frigeri et al. 1995b) kidney (Frigeri et al. 1995a), stomach (Frigeri et al. 1995b) and lung (Frigeri et al. 1995a) . The preliminary analysis of M1-AQP4 expression and AQP4 supramolecular organization in the kidney of OAP-null mice (Figure 4 Supplementary Information) has revealed an opposite trend with a downregulation of AQP4 tetramers associated with a massive reduction in M1-AQP4 expression which is undetectable by Western blot. This data indicates that the role of AQP4 isoforms into OAP assembly dynamics might depend on the cell type and that this mouse model could be useful to 
clarify these aspects.

AQP4-null mice totally lacking the AQP4 gene have largely been used to assess whether AQP4 plays a key role in organ physiology (Haj-Yasein et al. 2011; Ma et al. 1997; Verkman et al. 2006). However, though AQP4 deletion is associated with a number of functional defects, such as impaired hearing and olfaction ( $\mathrm{Li}$ and Verkman 2001), edema (Papadopoulos et al. 2004), epilepsy (Binder et al. 2004), stroke (Manley et al. 2000) and a mild concentrating defect in the kidney (Verkman et al. 2000), from the analysis of these phenotype studies it has emerged that deletion of AQP4 is not associated with any apparent phenotypic abnormality (Verkman et al. 2000). In this study, we have assessed that the OAP-null mouse growth is normal and no sign of suffering phenotype is revealed, in line with AQP4 KO mice totally lacking the AQP4 gene (Fan et al. 2005; Haj-Yasein et al. 2011; Ma et al. 1997). Moreover, no gross brain morphological abnormalities have been detected in line with previous data (Saadoun et al. 2009). However, future studies would need to be performed to ascertain whether and how the possibility to express the freely mobile M1AQP4 in OAP-null mice can affect glial cell migration/invasiveness, adhesion, polarization and apoptosis as is predictable based on the current literature (Simone et al. 2019; Smith et al. 2014).

To the best of our knowledge, this is the first study in which the concern over off-target cleavage in CRISPR-mediated genome editing has been considered important for potential corresponding offtarget biological effects in animal models. The entirely comparable results obtained between the two strains of OAP-null mice in terms of genotype-phenotype correlation give robustness to the main findings here shown and provide support to the affordability of CRISPR/Cas9 recently debated (Zhang et al., 2015; Schaefer et al., 2017; Iyer et al., 2018; Montoliu and Whitelaw, 2018).

In conclusion, this study demonstrates for the first time in vivo that the OAP supramolecular structure is a key feature characterizing the molecular identity of the AQP4 water channel and that 
the large majority of AQP4 is organized into OAPs under physiological condition, even independently of AQP4 polarized expression at astrocyte endfeet. The key information emerging from the characterization of the OAP-null mouse model here is also that OAP aggregation is essential to guarantee astrocytes a normal level of AQP4 expression in the plasma membrane and therefore AQP4 tetramers cannot be used by astrocytes as an alternative to OAPs without affecting AQP4 total expression levels. This is important in the light of astrocyte physiological and pathophysiological conditions in which OAP aggregation/disaggregation dynamics have been implicated, such as astrocyte migration and polarization, brain tumors and Neuromyelitis Optica. In particular, transitions from large OAPs to smaller OAPs or tetramers have been proposed to be regulated depending on cell physiological need (astrocyte migration or differentiation) without altering astrocyte permeability (Smith and Verkman, 2015). OAP dynamics can also influence the fate of glioma cells (Simone et al., 2019) toward cell migration or apoptosis and AQP4 aggregation into OAPs has a key role in Neuromyelitis Optica where NMO IgG autoantibodies can bind their autoantigen AQP4 only when aggregated into OAPs (Nicchia et al., 2009; Crane et al., 2011; Pisani et al., 2011). The OAP-null model could be useful for future studies aimed at identifying mechanisms underlying OAP biology and understanding the role of AQP4 aggregation dynamics under the physiological and pathophysiological circumstances mentioned above. It would also be a suitable model for assessing the role of the new emerging AQP4 intracellular and C-terminus extended AQP4 isoforms in cell biology and in OAP formation as well as for analyzing whether the alteration of AQP4 aggregation properties can be the basis of impaired organ function in those tissues in which AQP4 is expressed. We believe this study provides important clues to elucidate the cellular and molecular mechanism underpinning the role of astrocyte health and disease. 


\section{REFERENCES}

Amiry-Moghaddam M, Frydenlund DS, Ottersen OP. 2004a. Anchoring of aquaporin-4 in brain: molecular mechanisms and implications for the physiology and pathophysiology of water transport. Neuroscience 129(4):999-1010.

Amiry-Moghaddam M, Xue R, Haug FM, Neely JD, Bhardwaj A, Agre P, Adams ME, Froehner SC, Mori S, Ottersen OP. 2004b. Alpha-syntrophin deletion removes the perivascular but not endothelial pool of aquaporin-4 at the blood-brain barrier and delays the development of brain edema in an experimental model of acute hyponatremia. Faseb J 18(3):542-4.Badaut J, Fukuda AM, Jullienne A, Petry KG. 2014. Aquaporin and brain diseases. Biochim Biophys Acta - Gen Subj 1840:1554-1565.

Badaut J, Fukuda AM, Jullienne A, Petry KG. 2014. Aquaporin and brain diseases. Biochim Biophys Acta 1840(5):1554-65.

Benfenati V, Caprini M, Dovizio M, Mylonakou MN, Ferroni S, Ottersen OP, Amiry-Moghaddam M. 2011. An aquaporin-4/transient receptor potential vanilloid 4 (AQP4/TRPV4) complex is essential for cell-volume control in astrocytes. Proc Natl Acad Sci U S A 108:2563-2568.

Binder DK, Oshio K, Ma T, Verkman AS, Manley GT. 2004. Increased seizure threshold in mice lacking aquaporin-4 water channels. Neuroreport 15(2):259-62.

Bragg AD, Amiry-Moghaddam M, Ottersen OP, Adams ME, Froehner SC. 2006. Assembly of a perivascular astrocyte protein scaffold at the mammalian blood-brain barrier is dependent on $\alpha$-syntrophin. Glia 53:879-890.

Crane JM, Bennett JL, Verkman AS. 2009. Live cell analysis of aquaporin-4 M1/M23 interactions and regulated orthogonal array assembly in glial cells. J Biol Chem 284:35850-35860.

Crane JM, Lam C, Rossi A, Gupta T, Bennett JL, Verkman AS. 2011. Binding affinity and specificity of neuromyelitis optica autoantibodies to aquaporin-4 M1/M23 isoforms and 
orthogonal arrays. J Biol Chem 286:16516-16524.

De Bellis M, Pisani F, Mola MG, Rosito S, Simone L, Buccoliero C, Trojano M, Nicchia GP, Svelto M, Frigeri A. 2017. Translational readthrough generates new astrocyte AQP4 isoforms that modulate supramolecular clustering, glial endfeet localization, and water transport. Glia 65:790-803.

Fan Y, Zhang J, Sun XL, Gao L, Zeng XN, Ding JH, Cao C, Niu L, Hu G. 2005. Sex- and regionspecific alterations of basal amino acid and monoamine metabolism in the brain of aquaporin-4 knockout mice. J Neurosci Res 82:458-464.

Fenton RA, Moeller HB, Nielsen S, de Groot BL, Rutzler M. 2010. A plate reader-based method for cell water permeability measurement. Am J Physiol Renal Physiol 298(1):F224-30.

Frigeri A, Gropper MA, Turck CW, Verkman AS. 1995a. Immunolocalization of the mercurialinsensitive water channel and glycerol intrinsic protein in epithelial cell plasma membranes. Proc Natl Acad Sci U S A 92(10):4328-31.

Frigeri A, Gropper MA, Umenishi F, Kawashima M, Brown D, Verkman AS. 1995b. Localization of MIWC and GLIP water channel homologs in neuromuscular, epithelial and glandular tissues. J Cell Sci 108 ( Pt 9):2993-3002.

Furman CS, Gorelick-Feldman DA, Davidson KGV, Yasumura T, Neely JD, Agre P, Rash JE. 2003. Aquaporin-4 square array assembly: Opposing actions of M1 and M23 isoforms. Proc Natl Acad Sci U S A 100:13609-13614.

Haj-Yasein NN, Vindedal GF, Eilert-Olsen M, Gundersen GA, Skare Ø, Laake P, Klungland A, Thorén AE, Burkhardt JM, Ottersen OP, Nagelhus EA. 2011. Glial-conditional deletion of aquaporin-4 (Aqp4) reduces blood-brain water uptake and confers barrier function on perivascular astrocyte endfeet. Proc Natl Acad Sci U S A 108:17815-17820.

Hamann S, Kiilgaard JF, Litman T, Alvarez-Leefmans FJ, Winther BR, Zeuthen T. 2002. 
Measurement of cell-volume changes by fluorescence self-quenching. J Fluor 12: 139-145..

Hoddevik EH, Khan FH, Rahmani S, Ottersen OP, Boldt HB, Amiry-Moghaddam M. 2017. Factors determining the density of AQP4 water channel molecules at the brain-blood interface. Brain Struct Funct 222:1753-1766.

Iyer V, Boroviak K, Thomas M, Doe B, Riva L, Ryder E, Adams DJ. 2018. No unexpected CRISPR-Cas9 off-target activity revealed by trio sequencing of gene-edited mice. PLoS Genet 14.

Jin BJ, Rossi A, Verkman AS. 2011. Model of aquaporin-4 supramolecular assembly in orthogonal arrays based on heterotetrameric association of M1-M23 isoforms. Biophys J 100:2936-2945.

Jung JS, Prestont GM, Smith BL, Guggino WB, Agre P. 1994. Molecular structure of the water channel through aquaporin CHIP: The hourglass model. J Biol Chem 269:14648-14654.

Li J, Verkman AS. 2001. Impaired hearing in mice lacking aquaporin-4 water channels. J Biol Chem 276(33):31233-7.

Lisjak M, Potokar M, Rituper B, Jorgacevski J, Zorec R. 2017. AQP4e-Based Orthogonal Arrays Regulate Rapid Cell Volume Changes in Astrocytes. J Neurosci 37(44):10748-10756.

Lisjak M, Potokar M, Zorec R, Jorgačevski J. 2020. Indirect Role of AQP4b and AQP4d Isoforms in Dynamics of Astrocyte Volume and Orthogonal Arrays of Particles. Cells 9(3):735.

Lunde LK, Camassa LMA, Hoddevik EH, Khan FH, Ottersen OP, Boldt HB, Amiry-Moghaddam M. 2015. Postnatal development of the molecular complex underlying astrocyte polarization. Brain Struct Funct 220:2087-2101.

Ma T, Yang B, Gillespie A, Carlson EJ, Epstein CJ, Verkman AS. 1997. Generation and phenotype of a transgenic knockout mouse lacking the mercurial-insensitive water channel aquaporin-4. J Clin Invest 100:957-962.

Manley GT, Fujimura M, Ma T, Noshita N, Filiz F, Bollen AW, Chan P, Verkman AS. 2000. 
Aquaporin-4 deletion in mice reduces brain edema after acute water intoxication and ischemic stroke. Nat Med 6(2):159-63.

Moe SE, Sorbo JG, Sogaard R, Zeuthen T, Petter Ottersen O, Holen T. 2008. New isoforms of rat Aquaporin-4. Genomics 91(4):367-77.

Mola MG, Nicchia GP, Svelto M, Spray DC, Frigeri A. 2009. Automated Cell-Based Assay for Screening of Aquaporin Inhibitors. Anal Chem 81:8219-8229.

Mola MG, Sparaneo A, Gargano CD, Spray DC, Svelto M, Frigeri A, Scemes E, Nicchia GP. 2016. The speed of swelling kinetics modulates cell volume regulation and calcium signaling in astrocytes: A different point of view on the role of aquaporins. Glia 64:139-54.

Montoliu L, Whitelaw CBA. 2018. Unexpected mutations were expected and unrelated to CRISPRCas9 activity. Transgenic Res 27:315-319.

Nagelhus EA, Ottersen OP. 2013. Physiological Roles of Aquaporin-4 in Brain. Physiol Rev 93:1543-1562.

Neely JD, Christensen BM, Nielsen S, Agre P. 1999. Heterotetrameric composition of aquaporin-4 water channels. Biochemistry 38:11156-11163.

Neuhaus J. 1990. Orthogonal arrays of particles in astroglial cells: Quantitative analysis of their density, size, and correlation with intramembranous particles. Glia 3:241-251.

Nicchia GP, Cogotzi L, Rossi A, Basco D, Brancaccio A, Svelto M, Frigeri A. 2008. Expression of multiple AQP4 pools in the plasma membrane and their association with the dystrophin complex. J Neurochem 105:2156-2165.

Nicchia GP, Frigeri A, Liuzzi GM, Santacroce MP, Nico B, Procino G, Quondamatteo F, Herken R, Roncali L, Svelto M. 2000. Aquaporin-4-containing astrocytes sustain a temperature- and mercury-insensitive swelling in vitro. Glia 31:29-38.

Nicchia GP, Mastrototaro M, Rossi A, Pisani F, Tortorella C, Ruggieri M, Lia A, Trojano M, 
Frigeri A, Svelto M. 2009. Aquaporin-4 orthogonal arrays of particles are the target for neuromyelitis optica autoantibodies. Glia 57:1363-1373.

Nicchia GP, Nico B, Camassa LMA, Mola MG, Loh N, Dermietzel R, Spray DC, Svelto M, Frigeri A. 2004. The role of aquaporin-4 in the blood-brain barrier development and integrity: Studies in animal and cell culture models. Neuroscience 129:935-944.

Nielsen S, Nagelhus EA, Amiry-Moghaddam M, Bourque C, Agre P, Ottersen OR. 1997. Specialized membrane domains for water transport in glial cells: High- resolution immunogold cytochemistry of aquaporin-4 in rat brain. J Neurosci 17:171-180.

Noell S, Ritz R, Wolburg-Buchholz K, Wolburg H, Fallier-Becker P. 2012. An allograft glioma model reveals the dependence of Aquaporin-4 expression on the brain microenvironment. PLoS One 7:2-8.

Palazzo C, Buccoliero C, Mola MG, Abbrescia P, Nicchia GP, Trojano M, Frigeri A. 2019. AQP4ex is crucial for the anchoring of AQP4 at the astrocyte end-feet and for neuromyelitis optica antibody binding. Acta Neuropathol Commun 7:51.

Papadopoulos MC, Manley GT, Krishna S, Verkman AS. 2004. Aquaporin-4 facilitates reabsorption of excess fluid in vasogenic brain edema. Faseb J 18(11):1291-3.

Papadopoulos MC, Verkman AS. 2012. Aquaporin 4 and neuromyelitis optica. Lancet Neurol $11: 535-544$.

Pisani F, Mastrototaro M, Rossi A, Nicchia GP, Tortorella C, Ruggieri M, Trojano M, Frigeri A, Svelto M. 2011. Identification of two major conformational aquaporin-4 epitopes for neuromyelitis optica autoantibody binding. J Biol Chem 286:9216-24.

Rash JE, Davidson KGV, Yasumura T, Furman CS. 2004. Freeze-fracture and immunogold analysis of aquaporin-4 (AQP4) square arrays, with models of AQP4 lattice assembly. Neuroscience 129:915-934. 
Rossi A, Crane JM, Verkman AS. 2011. Aquaporin-4 Mz isoform: brain expression, supramolecular assembly and neuromyelitis optica antibody binding. Glia 59(7):1056-63.

Rossi A, Moritz TJ, Ratelade J, Verkman AS. 2012. Super-resolution imaging of aquaporin-4 orthogonal arrays of particles in cell membranes. J Cell Sci 125:4405-4412.

Rossi A, Pisani F, Nicchia GP, Svelto M, Frigeri A. 2010. Evidences for a Leaky Scanning Mechanism for the Synthesis of the Shorter M23 Protein Isoform of Aquaporin-4: Implication in orthogonal array formation and Neuromyelitis optica antibody interaction. J Biol Chem 285:4562-4569.

Saadoun S, Tait MJ, Reza A, Davies DC, Bell BA, Verkman AS, Papadopoulos MC. 2009. AQP4 gene deletion in mice does not alter blood-brain barrier integrity or brain morphology. Neuroscience 161(3):764-72.

Schaägger H, Cramer WA, von Jagow G. 1994. Analysis of molecular masses and oligomeric states of protein complexes by blue native electrophoresis and isolation of membrane protein complexes by two-dimensional native electrophoresis. Anal Biochem 217:220-230.

Schaefer KA, Wu WH, Colgan DF, Tsang SH, Bassuk AG, Mahajan VB. 2017. Unexpected mutations after CRISPR-Cas9 editing in vivo. Nat Methods 14:547-548.

Silberstein C, Bouley R, Huang V, Fang P, Pastor-Soler N, Brown D, Van Hoek AN. 2004. Membrane organization and function of M1 and M23 isoforms of aquaporin-4 in epithelial cells. Am J Physiol - Ren Physiol 287.

Simone L, Pisani F, Mola MG, De Bellis M, Merla G, Micale L, Frigeri A, Vescovi AL, Svelto M, Nicchia GP. 2019. AQP4 aggregation state is a determinant for glioma cell fate. Cancer Res 79:2182-2194.

Smith AJ, Jin B-J, Ratelade J, Verkman AS. 2014. Aggregation state determines the localization and function of M1- and M23-aquaporin-4 in astrocytes. J Cell Biol 204:559-573. 
Smith AJ, Verkman AS. 2015. Superresolution Imaging of Aquaporin-4 Cluster Size in AntibodyStained Paraffin Brain Sections. Biophys J 109:2511-2522.

Solenov E, Watanabe H, Manley GT, Verkman AS. 2004. Sevenfold-reduced osmotic water permeability in primary astrocyte cultures from AQP-4-deficient mice, measured by a fluorescence quenching method. Am J Physiol - Cell Physiol 286.

Verkman AS, Binder DK, Bloch O, Auguste K, Papadopoulos MC. 2006. Three distinct roles of aquaporin-4 in brain function revealed by knockout mice. Biochim Biophys Acta 1758(8):1085-93.

Verkman AS, Ratelade J, Rossi A, Zhang H, Tradtrantip L. 2012. Aquaporin-4: orthogonal array assembly, CNS functions, and role in neuromyelitis optica. Acta Pharmacol Sin 32(6):702-10.

Verkman AS, Yang B, Song Y, Manley GT, Ma T. 2000. Role of water channels in fluid transport studied by phenotype analysis of aquaporin knockout mice. Exp Physiol 85 Spec No:233S$241 \mathrm{~S}$.

Verkman AS, Rossi A, Crane JM. 2012. Live-cell imaging of aquaporin-4 supramolecular assembly and diffusion. In: Methods in Enzymology. Vol. 504. Academic Press Inc. p 341-354.

Wilcock DM, Vitek MP, Colton CA. 2009. Vascular amyloid alters astrocytic water and potassium channels in mouse models and humans with Alzheimer's disease. Neuroscience 159:10551069.

Yang B, Verkman AS. 1997. Water and glycerol permeabilities of aquaporins 1-5 and MIP determined quantitatively by expression of epitope-tagged constructs in Xenopus oocytes. $\mathrm{J}$ Biol Chem 272:16140-6.

Zhang XH, Tee LY, Wang XG, Huang QS, Yang SH. 2015. Off-target effects in CRISPR/Cas9mediated genome engineering. Mol Ther - Nucleic Acids 4:e264. 


\section{FIGURE LEGENDS}

Figure 1. Generation of OAP-null mouse using the CRISPR/Cas9 system. A) Schematic representation of the targeted exon 1 of mouse AQP4 on chromosome 18. Coding exons are shown as solid black bars and are interspaced by non-coding sequences represented with thin lines. Two overlapping gRNAs, gRNA1 (red line) and gRNA2 (blue line), were used to target different AQP4 sequences of the wildtype allele. The proto spacer-adjacent motif (PAM) sequences are underlined for each gRNA (PAM1 for gRNA1 and PAM2 for gRNA2) while the single base (G) to be mutated is highlighted by an asterisk in the wildtype allele before targeting. The green scissors indicate the Cas9 cleavage site. The mutated base "C" $(\mathrm{G} \rightarrow \mathrm{C})$ is highlighted in red in the mutant alleles obtained after targeting. The mutated allele obtained with gRNA1 displayed an additional silent mutation (in grey) at the PAM sequence. B) The genome of wildtype mice with C57BL/6 genetic background was edited by injecting Cas9 (in green), donor oligo sequences containing desired mutations and gRNAs (gRNA1 in red and gRNA2 in blue) in fertilized eggs. C) Sequence analysis of the mutant alleles in heterozygous mice. The desired mutation $\mathrm{G} \rightarrow \mathrm{C}$ (red arrows) in the $A Q P 4$ gene was confirmed in both gRNA1 and gRNA2 strains of KI mice. The silent mutation $(\mathrm{C} \rightarrow \mathrm{A})$ at the PAM sequence is shown in grey for gRNA1 mutant allele.

Figure 2. Analysis of AQP4 mRNA by qPCR and AQP4 assemblies by BN-PAGE and BN/SDS-PAGE in WT and OAP-null brain and cerebellum. A) Real time PCR analysis of relative AQP4 mRNA levels in the brain and cerebellum from WT and OAP-null mice. AQP4 mRNA levels were significantly higher in the cerebellum compared with the brain but there were no significant differences between tissues from WT and OAP-null mice (unpaired Student's $t$-test: $* * * P<0.001, \mathrm{n}=3$. B) Left: membrane proteins extracted from the brain and cerebellum of OAPnull.1, OAP-null.2 and WT mice were separated under native conditions on 3-9\% acrylamide gels (BN-PAGE) and then subjected to immunoblot using AQP4 antibodies against all AQP4 isoforms 
(AQP4 $\mathrm{Ab})$. Tissues from AQP4 KO mice $(\mathrm{KO})$ were used as negative controls. In WT tissues, AQP4 tetramers (arrow) and several different pools corresponding to different sized AQP4 assemblies (arrowheads) are visualized. In contrast, in OAP-null tissues only the smaller AQP4 pool corresponding to AQP4 tetramers is visualized. Right: Bar graph summarizing the densitometric analysis, shown as arbitrary units (A.U.), performed on AQP4 tetramers indicated by the arrow in the tissues, as indicated. Data from OAP null.1 and OAP-null.2 have been pooled together since no significant difference was found between the two strains (unpaired Student's $t$ test: $\left.{ }^{*} P<0.05,{ }^{*} P<0.01, \mathrm{n}=3-6\right)$. Data in $\left.\mathbf{A}\right)$ and $\left.\mathbf{B}\right)$ are expressed as means \pm standard error of the mean (SEM). C) Two-dimensional analysis of AQP4 assemblies in WT and OAP-null brain and cerebellum. After being separated under native conditions in 3-9\% acrylamide gels (BN-PAGE, first dimension), membrane proteins were subjected to a second dimension performed under denaturing condition (SDS-PAGE), using a $13 \%$ acrylamide gel. Immunoblot was performed using AQP4 Ab. Tissues from AQP4 KO (KO) mice were used as negative controls. AQP4 tetramers are indicated by vertical arrows and AQP4 assemblies by arrowheads. M1-AQP4 (M1) and M1AQP4ex (M1ex) are indicated by horizontal arrows.

Figure 3. Western blot analysis of M1-AQP4 and M23-AQP4 expression in WT and OAP-null brain and cerebellum. A) Left: Western blot analysis of M1-AQP4 (M1) and M23-AQP4 (M23) expression in the brain and cerebellum from WT, OAP-null (OAP-null.1 and OAP-null.2) and AQP4 KO (KO) mice, performed using AQP4 Ab and antibodies able to recognize the M1-AQP4 isoform $(\mathrm{M} 1 \mathrm{Ab})$ selectively. The asterisk indicates a non specific band recognized by M1 Ab in all the brain and cerebellum tissues analyzed, including those from AQP4 KO mice, differing in size between brain and cerebellum. In the Western blot performed with AQP4 Ab, a ten-times (10X) or a 5 times (5X) higher concentration of OAP null tissues was loaded to visualize the correct expression of the M1-AQP4 isoform. In the 5X and 10X samples from OAP-null mice, the M1AQP4ex isoform (M1ex) and a band presumably corresponding to AQP4f (arrowhead) are 
indicated. Right: bar graphs summarizing the densitometric analysis of M1-AQP4 (M1/ Coomassie Blue, shown in \%) quantified from Western blot performed using M1 Ab in brain and cerebellum, as indicated. Coomassie Blue staining of total proteins was used as an internal control for protein loading (unpaired Student's $t$-test: $* * * P<0.001, \mathrm{n}=6$ ). Data are expressed as means $\pm \mathrm{SEM}$. B) Schema showing the effect of M23-AQP4 deletion on its molecular partner M1-AQP4 and on AQP4 tetramer expression based on the data shown in Figure 2B and Figure 3A.

Figure 4. Analysis of AQP4 assemblies in WT and OAP-null astrocyte primary cultures by BN-PAGE, Western blot, and water transport assay. A) Left: Membrane proteins extracted from OAP-null and WT astrocytes were separated under native conditions in 3-9\% acrylamide gels (BNPAGE) and then subjected to immunoblot analysis using AQP4 Ab. Astrocytes from AQP4 KO mice $(\mathrm{KO})$ were used as negative controls. AQP4 tetramers are indicated by an arrow and AQP4 assemblies by arrowheads. Right: bar graph summarizing the densitometric analysis, shown as arbitrary units (A.U.) performed on AQP4 tetramer in WT and OAP-null astrocytes (unpaired Student's $t$-test: $* * P<0.01, \mathrm{n}=3)$. B) Left: Western blot analysis of AQP4 expression in astrocytes from WT, OAP-null and AQP4 KO (KO) mice, as indicated, performed using AQP4 Ab and M1 Ab. Right: bar graph summarizing the densitometric analysis of M1-AQP4 (M1/ Coomassie Blue, shown in \%) quantified from Western blot performed using M1 Ab in WT and OAP-null astrocyte primary cultures. Coomassie Blue staining was used as an internal control for protein loading (unpaired Student's $t$-test: $* * * P<0.001, \mathrm{n}=3$ ). C) Left: Functional analysis for AQP4 mediated water transport performed with the calcein quenching assay using the Flex Station multiplate reader. The typical shrinkage kinetics induced by osmotic shocks (hypertonic solution) and recorded from calcein-AM loaded WT, OAP-null and AQP4 KO (KO) astrocytes is shown. Middle: Bar graph of the mean \pm SEM values of time constants obtained by fitting an exponential curve to the shrinkage of cells after exposure to 360 mOsm solution. Right: Bar graph showing the mean \pm 
SEM values of the maximal amplitude of astrocyte shrinkage (ANOVA followed by NewmanKeuls test: $\left.* P<0.05,{ }^{*} P<<0.01 ; * * * P<0.001, \mathrm{n}=3\right)$.

Figure 5. Analysis of AQP4 assemblies in WT and OAP-null astrocyte primary cultures by gSTED microscopy. A) Left and middle panels: representative images of WT, OAP-null and KO astrocytes, stained with AQP4 Ab (green) and GFAP Ab (red) obtained by confocal microscopy. Right panels: boxed yellow areas are shown at higher magnification with AQP4-aggregates imaged by confocal (upper insets) and gSTED (lower insets) microscopy, as indicated. Scale bars: $10 \mu \mathrm{m}$ (whole-cell); $500 \mathrm{~nm}$ (confocal and STED insets). B) AQP4-aggregate surface $\left(\mathrm{nm}^{2}\right)$ frequency histogram of WT (left) and OAP-null (right) astrocytes, showing the number of analyzed clusters plotted against the cluster dimension. C) Differences in AQP4-aggregate median size between genotypes. In the box plot, the box represents the interquartile range (IQR), the central line is the median, the plus sign ' + ' identifies the mean values, and the whiskers extend from the minimum to the maximum values (Shapiro-Wilk normality test $* * * \quad \mathrm{P}<0.001$ followed by unpaired MannWhitney test: $* * * P<0.001, \mathrm{n}=3$ ). D) Quantification of AQP4-aggregate density expressed as the number of STED-identified clusters normalized to the cell surface area. Data are presented as mean \pm SEM (unpaired Student T-test: $* * * P<0.001, \mathrm{n}=3$ ).

Figure 6. AQP4 distribution and morphological analysis on OAP-null and WT brain and cerebellum. AQP4 localization by immunofluorescence, immunoperoxidase and trichrome stain of brain cortex and cerebellum sections prepared from WT and OAP-null mice. Arrows indicate AQP4 staining which is clearly visible and specific in the WT tissues and very faint and diffuse in OAPnull tissues. CX, cortex; M, molecular cell layer; G, granular cell layer. Scale bar: $100 \mu \mathrm{m}$. 
Figure 7. AQP4 immunogold labeling in OAP-null and WT cortex. Electron micrographs showing AQP4 immunogold labeling in cortex of WT (A and C) and OAP-null (B and D) mice. No AQP4 immnunogold labeling is visible at the perivascular astrocytic (B) membrane and only a few immunogold particles are present at the subpial endfoot (D) of the OAP-null mouse cortex. A and B are from perivascular membranes (arrows), while C and D are from subpial membranes (arrows). L $=$ lumen, $\mathrm{E}=$ endothelium, $\mathrm{P}=$ pericyte, asterisk = basal membrane. Scale bars $(\mathrm{A}, \mathrm{B}: 500 \mathrm{~nm} ; \mathrm{C}$, $\mathrm{D}: 1 \mu \mathrm{m})$.

Figure 8. AQP4 immunogold labeling o in OAP-null and WT cerebellum. Electron micrographs showing AQP4 immunogold labeling in cerebellum of WT (A, C, E) and OAP-null mice (B, D, F). Note the strong reduction in the perivascular and subpial AQP4 immunogold labeling of the OAPnull mice compared to the WT. Immunogold particles indicating AQP4 labeling in astrocyte processes in the white matter of cerebellum are shown with double-headed arrows. A and B are from perivascular membranes (arrows) in the granule layer. C and D are from perivascular membranes (arrows) in the white matter. $\mathrm{E}$ and $\mathrm{F}$ are from subpial membranes (arrows). L = lumen, $\mathrm{E}=$ endothelium, $\mathrm{P}=$ pericyte, $\mathrm{Ax}=$ axon, $\mathrm{my}=$ myelin, doubled arrows $=$ other astrocyte membranes, asterisk $=$ basal membrane, arrowheads $=$ collagen fibers of pial mater. Scale bars $(\mathrm{C}$ : $500 \mathrm{~nm} ; \mathrm{A}, \mathrm{B}, \mathrm{D}-\mathrm{F}: 1 \mu \mathrm{m})$

Figure 4 Supplementary Information. Analysis of AQP4 assemblies and M1-AQP4 and M23AQP4 expression by BN-PAGE, BN/SDS-PAGE and Western blot in WT and OAP-null kidney plasma membrane vesicles. Membrane proteins were extracted from plasma membrane vesicles prepared from the kidney medulla of OAP-null and WT mice as follows: mouse tissue was cut into small pieces and homogenized with a Dounce apparatus in five volumes of ice-cold Homogenizing Buffer (250 mM Sucrose; $10 \mathrm{mM}$ Tris-HCl, $\mathrm{pH}$ 7,5) added with protease inhibitor cocktail. Homogenates were centrifuged at $4,000 \mathrm{xg}$ for 10 minutes at $4^{\circ} \mathrm{C}$. After centrifugation, 
pellets were discarded and supernatants collected and centrifuged at $17,000 \mathrm{xg}$ for 45 minutes at $4^{\circ} \mathrm{C}$ to obtain a low-speed membrane vesicles-enriched pellet. The resulting pellets were resuspended in seven volumes of BN-PAGE lysis buffer added with protease inhibitor for one hour on ice and vortexed every 10 minutes. Vesicle lysates were then centrifuged at $21,000 \mathrm{xg}$ for one hour at $4^{\circ} \mathrm{C}$. The supernatant was collected and the protein content was measured using the $\mathrm{BCA}^{\mathrm{TM}}$ Protein Assay Kit (http://www.thermoscientific.com).

A) Extracted proteins were separated under native conditions on 3-9\% acrylamide gels (BN-PAGE) and then subjected to immunoblot using AQP4 antibodies against all AQP4 isoforms (AQP4 Ab). AQP4 tetramers are indicated by the arrow and pools corresponding to AQP4 assemblies are indicated by arrowheads. Right: Bar graph summarizing the densitometric analysis, shown as arbitrary units (A.U.), performed on AQP4 tetramers indicated by the arrow in the tissues, as indicated (unpaired Student's $t$-test, ${ }^{*} P<0.05, \mathrm{n}=3$ ). Data are expressed as means \pm standard error of the mean (SEM). B) Two-dimensional analysis of AQP4 assemblies in WT and OAP-null kidney plasma membrane vesicles. After being separated under native conditions in 3-9\% acrylamide gels (BN-PAGE, first dimension), membrane proteins were subjected to a second dimension performed under denaturing condition (SDS-PAGE), using a $13 \%$ acrylamide gel. Immunoblot was performed using AQP4 Ab. AQP4 tetramers are indicated by vertical arrows and AQP4 assemblies by arrowheads. C) Western blot analysis of M1-AQP4 (M1) and M23-AQP4 (M23) expression in the vesicles from WT and OAP-null mice, performed using AQP4 Ab. Brain tissues were used as control. A seven-times (7X) or three-times (3X) higher concentration of OAP null tissues was loaded to visualize the expression of the M1-AQP4 isoform.

The results show that no tetramer upregulation occurs in OAP-null kidneys and that M1-AQP4 expression is massively reduced and undetectable by Western blot even using a seven-times higher concentration of protein sample compared with the WT. 
Running Title: OAP-null mouse model

\section{Orthogonal arrays of particle assembly are essential for normal}

\section{Aquaporin-4 expression level in the brain}

Manuela De Bellis ${ }^{1, *}$, Antonio Cibelli,,*, Maria Grazia Mola,,*, Francesco Pisani ${ }^{1}$, Barbara Barile $^{1}$, Maria Mastrodonato ${ }^{2}$, Shervin Banitalebi ${ }^{3}$, Mahmood Amiry-Mogghaddam $^{3}$, Pasqua $^{2}$ Abbrescia $^{4}$, Antonio Frigeri ${ }^{4}$, Maria Svelto ${ }^{1}$, Grazia Paola Nicchia ${ }^{1, \#}$

${ }^{1}$ Department of Biosciences, Biotechnologies and Biopharmaceutics, University of Bari Aldo Moro, Via Orabona 4, 70125 Bari, Italy.

${ }^{2}$ Department of Biology, University of Bari Aldo Moro, Via Orabona 4, 70125 Bari, Italy.

${ }^{3}$ Department of Molecular Medicine, Division of Anatomy, University of Oslo, P.O Box 1105 Blindern 0317 Oslo, Norway.

${ }^{4}$ School of Medicine, Department of Basic Medical Sciences, Neuroscience and Sense Organs, University of Bari Aldo Moro, Policlinico, Piazza G. Cesare 11, 70124Bari, Italy.

*Manuela De Bellis, Antonio Cibelli and Maria Grazia Mola should be considered joint first author

\#Grazia Paola Nicchia is the corresponding author (email: graziapaola.nicchia@uniba.it)

Acknowledgments This work was supported by a grant from the Italian Ministry of the University and Research (FIRB-Futuro in Ricerca RBFR12SJA8) to GPN. The authors would like to thank Richard Lusardi for his expert assistance in revising the English of the article and Gaetano Devito for excellent technical assistance.

Conflict of interest statement: The authors do not have any potential sources of conflict of interest.

Data Availability Statement: The authors confirm that the data supporting the findings of this study are available within the article.

\section{Word count}

Manuscript 10718; Abstract 238; Introduction 969; Materials and Methods 2261; Results 1637;

Discussion 2011; References 1864

\section{MAIN POINTS}

1) Orthogonal arrays of particle (OAP) assembly is essential for normal Aquaporin-4 expression level in the brain. 2) Most brain AQP4 is organized into OAPs under physiological conditions. 


\begin{abstract}
Astrocyte endfeet are endowed with Aquaporin-4 (AQP4)-based assemblies called Orthogonal Arrays of Particles (OAPs) whose function is still unclear. To investigate the function of OAPs and of AQP4 tetramers, we have generated a novel "OAP-null" mouse model selectively lacking the OAP forming M23-AQP4 isoform. We demonstrated that AQP4 transcript levels were not reduced by using qPCR. BN/SDS-PAGE and Western blot performed on OAP-null brain and primary astrocyte cultures showed the complete depletion of AQP4 assemblies, the selective expression of M1-AQP4-based tetramers and a substantial reduction in AQP4 total expression level. Fluorescence quenching and super-resolution microscopy experiments showed that AQP4 tetramers were functionally expressed in astrocyte plasma membrane and their dimensions were reduced compared to wild-type assemblies. Finally, as shown by light and electron microscopy, OAP depletion resulted in a massive reduction in AQP4 expression and a loss of perivascular AQP4 staining at astrocyte endfeet, with only sparse labelling throughout the brain areas analysed. Our study relies on the unique property of AQP4 to form OAPs, using a novel OAP-null mouse model for the first time, to show that 1) AQP4 assembly is essential for normal AQP4 expression level in the brain and 2) most of AQP4 is organized into OAPs under physiological conditions. Therefore, AQP4 tetramers cannot be used by astrocytes as an alternative to OAPs without affecting AQP4 expression levels, which is important in the physiological and pathological conditions in which OAP aggregation/disaggregation dynamics have been implicated.
\end{abstract}

\title{
KEYWORDS
}

Astrocytes, Aquaporin-4, Orthogonal Arrays of Particles, Assembly, Tetramers, CRISPR/Cas9, Brain 


\section{INTRODUCTION}

One of the key functions of astrocytes is the control of osmotic and volume physiological parameters guaranteed by the water channel protein Aquaporin-4 (AQP4) (Badaut et al. 2014; Benfenati et al., 2011; Mola et al., 2016). AQP4 is mainly localized at astrocytic endfeet (Nielsen et al., 1997) at the interface between the Central Nervous System (CNS) and liquid compartments (Amiry-Moghaddam et al. 2004b; Hoddevik et al. 2017). In addition, there is a minor "perisynaptic pool" of AQP4 which has been proposed to control the extracellular space volume challenged by neuronal activity (Nagelhus and Ottersen, 2013).

The peculiarity of AQP4 is its plasma membrane organization into large well-ordered assemblies called "square arrays" or "orthogonal array of particles (OAPs)", visible by Freeze Fracture Electron Microscopy (FFEM). Interestingly, OAP alterations have been observed in different pathological conditions such as brain tumors, ischemia and Alzheimer's disease (Neuhaus, 1990; Wilcock et al., 2009; Noell et al., 2012). Moreover, the interest in OAP studies has recently increased since the discovery of their involvement in the pathogenesis of Neuromyelitis Optica (NMO), a CNS autoimmune channelopathy whose autoantibodies are only able to attack their antigen AQP4 if arranged in OAPs (Nicchia et al., 2009a; Crane et al., 2011; Papadopoulos and Verkman, 2012).

The molecular nature of OAPs resides in the distinct characteristic of AQP4 of being expressed as different similar isoforms by two operating mechanisms called leaky scanning (Rossi et al., 2010) and translational read-through (De Bellis et al., 2017; Palazzo et al., 2019), able to produce isoforms with a shorter N-terminus or with a longer C-terminus, respectively, as well as by alternative splicing (Moe et al. 2008). 
M1-AQP4 and M23-AQP4 are more abundant and the very well characterized AQP4 isoforms synthetized from two in-frame translation initiation sites, Met-1 for M1-AQP4 and Met-23 for M23-AQP4 (Furman et al. 2003). Two additional AQP4 isoforms have recently been identified, M1-AQP4ex and M23-AQP4ex, each having an extension at the C-terminus (De Bellis et al., 2017; Palazzo et al., 2019). By alternative splicing, six cDNA AQP4 isoforms, named AQP4a-f, can be expressed in rats (Moe et al. 2008), having a different N-terminus and sharing a common Cterminus. AQP4a and AQP4c correspond to the two classical M1- and M23-AQP4 isoforms, respectively.

The major M23- and M1-AQP4 isoforms are very well characterized (Furman et al., 2003; Smith et al., 2014) for their role in OAP formation. When transfected separately, they show similar water permeability (Neely et al., 1999; Silberstein et al., 2004) but different aggregation properties. In particular, M23-AQP4 forms OAPs while M1-AQP4 exists mainly as individual tetramers (Rash et al., 2004). However, when co-expressed, M1-AQP4 can assemble into OAPs together with M23AQP4 as hetero-tetramers, reducing OAP size and affecting their shape (Neely et al., 1999; Crane et al., 2009; Rossi et al., 2012). Interestingly, OAPs have an M23-AQP4-enriched core surrounded by an M1-AQP4-enriched periphery. It thus seems likely that the hydrophobic intermolecular interactions occurring between the shorter N-terminus of M23-AQP4 provide the adhesive properties stabilizing the OAPs, while the additional N-terminus of M1-AQP4 disturbs these interactions, therefore interrupting the increase in OAP dimension (Rossi et al., 2012). Interestingly, an active role of $\mathrm{AQP} 4 \mathrm{e}$ in the regulation of OAP structural dynamics has been recently demonstrated with AQP4e affecting the cell abundance of OAPs in hypoosmotic conditions (Lisjak et al. 2017). However, AQP4e expression, documented in rat, is not predicted in mouse and human due to the presence of a STOP codon upstream of the M1 start codon (Rossi et al. 2011). Moreover, a role in regulating OAP size has been proposed for AQP4ex isoforms (Palazzo et al. 2019). 
It has been proposed that OAP assembly/disassembly dynamics has an impact on astrocytes in health and disease (Amiry-Moghaddam et al. 2004a; Simone et al. 2019; Smith et al. 2014; Verkman et al. 2012). Although M1-AQP4 and M23-AQP4 are always coexpressed in vivo, studies performed in cell cultures transfected with the two isoforms separately have indicated different functional roles of M1-AQP4 based tetramers and M23-AQP4 based OAPs. M1-AQP4 based tetramers or small M1-AQP4 enriched OAPs are mobile in the plasma membrane and have been proposed to rapidly diffuse to the front edge of migrating astrocytes (Smith et al., 2014). In contrast, large stable M23-AQP4 enriched OAPs, are proposed to support the opposite function of cell adhesion as they are important for AQP4 polarization at glial endfoot microdomains (AmiryMoghaddam et al., 2004; Verkman et al., 2012; Smith et al., 2014). Moreover, AQP4 aggregation has recently been shown to influence plasma membrane dynamics as well as to alter cell proliferation, invasiveness, migration, and apoptotic potential in glioma cells (Simone et al., 2019). This indicates a new perspective on the role of AQP4 in brain tumors, which is not necessarily associated with edema formation but rather with AQP4 tetramer and OAP dynamics and their link with the actin cytoskeleton.

Based on the important role of AQP4 aggregation into OAPs in the physiology and pathophysiology of CNS glial cells, we have here used the CRISPR/Cas-9 technology to generate a new mouse model selectively lacking the OAP forming isoform M23-AQP4 and therefore called OAP-null mouse. Given the concerns about the offtarget genes potentially targeted by the use of short gRNAs, we produced two different strains of mice carrying the same mutation but using two diverse and independent strategies. The unaltered expression of AQP4 mRNA, the functional expression of AQP4 tetramers and the inability to form supramolecular assemblies conducible to OAPs have been validated in the two mice strains. Biochemical combined with functional assays and light, super-resolution and electron microscopy experiments have provided important information on the role of AQP4 tetramers and their aggregation into OAPs in the brain under 
normal conditions. We believe this study provides a better mechanistic understanding of the unique property of AQP4 to aggregate into OAPs using a novel OAP-null mouse model for the first time. 


\section{MATERIALS AND METHODS}

Ethics statement. In this study no experiments were performed on live animals. Experiments were performed according to the European directive on animal use for research and the Italian law on animal care. The protocols were approved by the Italian Ministry of Health (Protocol no. 710/2017PR and 571/2018-PR). All experiments were designed to minimize the number of animals used and animal suffering. Mice were maintained under a 12-h dark to light cycle, at constant room temperature (RT) and humidity, with food and water provided ad libitum, and supplied with environmental enrichment materials such as toys and shelters.

Animals. OAP-null mice harboring the M23I point mutation were generated on the C57BL/6J background by Cyagen Biosciences (Santa Clara, CA, USA) using CRISPR/Cas9-based targeting and homology-directed repair. The details of the genome editing approach are described in the Results section. In founder lines, the lack of mutations in off-target genes was verified by PCR amplification and sequencing of selected candidate genes. Intercrossing of heterozygous mice yielded healthy offspring at Mendelian ratios. AQP4 knockout (KO) mice with a CD1 genetic background and age-matched CD1 mice, used as WT mice, were kindly provided by Dr. Hu (Nanjing Medical University, China). Genotyping was performed on tail DNA using standard protocols.

Antibodies. The primary antibodies used were: goat polyclonal anti-AQP4 (C-19, Santa Cruz, Dallas, TX, USA) and rabbit polyclonal anti-AQP4 (H-80, Santa Cruz, Dallas, TX, USA) both diluted 1:400 for immunofluorescence and 1:500 for immunoblot analysis; rabbit anti-AQP4 (A5971, Sigma-Aldrich, Saint Louis, MO, USA) at 1:400 dilution for electron microscopy, and mouse monoclonal anti-GFAP (clone G-A-5, Millipore, Burlington, MA, USA) at 1:500 for 
immunofluorescence. An affinity-purified rabbit polyclonal antibody to a predicted antigen (MSDRAAARRWGKC) within the mouse M1-AQP4 specific sequence was prepared by GenScript (Leiden, Netherlands) and diluted 1:1000 for immunoblot analysis. The following secondary antibodies (all from Santa Cruz, Dallas, TX, USA) were used for Western blot diluted to 1:5000: donkey anti- goat IgG-horse- radish peroxidase (HRP); goat anti-rabbit IgG-HRP. The secondary antibodies used for immunofluorescence were: Alexa Fluor594 donkey anti-mouse, Alexa Fluor488 donkey anti-rabbit and Alexa Fluor488 donkey anti-goat (all from Thermo, Waltham, MA, USA) diluted 1:1000. The secondary antibody used for immunohistochemistry was goat anti-rabbit HRPconjugate diluted 1:100 whereas for immunogold electron microscopy it was goat anti-rabbit antibody coupled to $15 \mathrm{~nm}$ colloidal gold particles (1:20, Abcam, Cambridge, UK).

Astrocyte primary cell culture. Mouse astrocyte primary cultures were prepared from newborn pups as previously described (Nicchia et al., 2000). Cells were cultured in DMEM-Glutamax medium supplemented with $10 \%$ fetal bovine serum (FBS), $100 \mathrm{U} / \mathrm{ml}$ penicillin and $100 \mathrm{mg} / \mathrm{ml}$ streptomycin, and maintained at $37^{\circ} \mathrm{C}$ in a $5 \% \mathrm{CO}_{2}$ incubator.

Brain, cerebellum and astrocyte primary culture protein lysates. Brains were dissolved in seven volumes of $\mathrm{BN}$ buffer (1\% Triton X-100, $12 \mathrm{mM} \mathrm{NaCl,} 500 \mathrm{mM}$ 6-aminohexanoic acid, $20 \mathrm{mM}$ Bis-Tris, pH 7.0, 2 mM EDTA, 10\% glycerol) added with Protease Inhibitor Cocktail (Roche, Indianapolis, IN, USA). A confluent layer of astrocyte primary cultures was washed once with icecold PBS, harvested with a cell scraper, and lysed into seven volumes of BN buffer plus Protease Inhibitor Cocktail (Roche, Indianapolis, IN, USA). After $1 \mathrm{~h}$ incubation on ice the samples were then centrifuged at $21,000 \mathrm{Xg}$ for $30 \mathrm{~min}$ at $4{ }^{\circ} \mathrm{C}$. The supernatants were collected, and the total protein content was calculated using the BCA Protein Assay Kit (Thermo, Waltham, MA, USA). 
Western blotting. Protein lysates were dissolved in Laemmli Sample Buffer (Bio-Rad, Hercules, CA, USA) added with $50 \mathrm{mM}$ dithiothreitol, heated to $37^{\circ} \mathrm{C}$ for $10 \mathrm{~min}$, resolved in a $13 \%$ polyacrylamide gel, and transferred onto polyvinylidenedifluoride (PVDF) membranes (Immobilon PVDF; Millipore, Burlington, MA, USA) for immunoblot analysis. Membranes containing the blotted proteins were blocked with $5 \%$ nonfat dried milk, incubated with primary antibodies for 2 h, washed and incubated with HRP-conjugated secondary antibodies. After washing, reactive proteins were revealed using an enhanced chemiluminescent detection system (ECL-Plus; Pierce Euroclone, Pero, MI, Italy) and visualized on a Chemi-Doc imaging system (Bio-Rad, Hercules, CA, USA). Images were recorded and data analyzed with Image lab software (Bio-Rad, Hercules, CA, USA).

Blue Native (BN)-PAGE and two-dimensional BN/SDS-PAGE. $50 \mu \mathrm{g}$ of protein sample were mixed with 5\% Coomassie Blue-250 and loaded onto a polyacrylamide native gradient gel (3-9\%) (Schaägger et al., 1994). The running buffers were as follows: anode buffer (25 mM imidazole, $\mathrm{pH}$ 7) and blue cathode buffer (50 mMtricine, $7.5 \mathrm{mM}$, imidazole, 0.02\% Coomassie Blue G-250, $\mathrm{pH}$ 7). Electrophoresis was performed at $6 \mathrm{~mA}$ and stopped when the tracking line of the Coomassie Blue G-250 dye had left the edge of the gel. Proteins were blotted onto PVDF membranes (Millipore, Burlington, MA, USA) for immunoblot analysis or alternatively, the lanes were used for the second dimension (2D). For the 2D BN/SDS-PAGE analysis, lanes from the first dimension were cut into individual strips and equilibrated in denaturing buffer $(1 \%$ SDS and $1 \%$ mercaptoethanol) for $1 \mathrm{~h}$ at RT and placed in a 13\% 2D SDS-polyacrylamide gel with the same thickness. At the end of the run, the gel was blotted onto a PVDF membrane (Millipore, Burlington, MA, USA) for Western blot analysis performed as described above. 
Immunofluorescence on tissues sections. For brain sections, the tissue was isolated, fixed in $4 \%$ PFA solution overnight $(\mathrm{O} / \mathrm{N})$ at $4{ }^{\circ} \mathrm{C}$, washed in PBS, immersed in $30 \%$ sucrose solution and frozen at $-80^{\circ} \mathrm{C}$. For immunofluorescence experiments, sections of $10 \mu \mathrm{m}$ thickness were cut on a cryostat (CM 1900; Leica, Wetzlar, Germany) at $-20^{\circ} \mathrm{C}$. After blocking with $0.1 \%$ Gelatin in PBS, sections were incubated with primary antibodies $\mathrm{O} / \mathrm{N}$ at $4{ }^{\circ} \mathrm{C}$ or for $1 \mathrm{~h}$ at $\mathrm{RT}$ in blocking solution. After 30 min of washing, sections were incubated with secondary antibodies for $1 \mathrm{~h}$, washed again for 15 min in PBS and mounted with Mowiol or in PBS-glycerol (1:1) pH 8.0, containing 1\% npropylgallate. Immunostained tissues were observed with a Leica DM6000B microscope equipped with HC PL Fluotar 10x and 40x objectives. In all experiments, no-primary and no-secondary antibody controls were run in parallel. No specific staining was observed in these controls.

Stimulated emission depletion (STED) microscopy and data analysis. Immunofluorescence for gated STED (gSTED) was performed as reported in the Quick Guide to the STED Sample Preparation (http://www.leica- microsystems.com). Briefly, the cells were washed with PBS, fixed with 2\% PFA (Sigma-Aldrich, Saint Louis, MO, USA) in PBS for $10 \mathrm{~min}$ and permeabilized with $0.3 \%$ Triton X-100 (Sigma-Aldrich, Saint Louis, MO, USA) for $10 \mathrm{~min}$. Cells were saturated with 2\% BSA (Sigma-Aldrich, Saint Louis, MO, USA) in PBS, incubated with primary antibodies in 2 \% BSA in PBS, washed and incubated with secondary Alexa Fluor-conjugated antibodies (Thermo, Waltham, MA, USA) in 2\% BSA in PBS. After three washings with PBS cells were finally mounted with Mowiol. A Leica TCS SP8 STED 33 microscope and Leica LASX software were used for image acquisition and analysis (Leica Microsystems CMS GmbH, Wetzlar, Germany). A Leica HC PL APO 1003/1.40 Oil STED White objective and Type F Immersion liquid with a refractive index of 1.5 were used. Excitation of the Alexa Fluor488 dye was achieved with a continuous wave at $488 \mathrm{~nm}$ using a diode laser with a maximum light output in the focal plane of 10 
$\mathrm{mW}$ (NKT Photonics supercontinuum laser). Depletion was performed using a fiber laser with a continuous wave of $592 \mathrm{~nm}$ and a maxim light output in the focal plane of $<0.5 \mathrm{~mW}$ (Laser Quantum). Multiple random regions of interest (ROI) $(\sim 20 \times 20 \mu \mathrm{m}$ sized) were taken using extra optical zoom (5-7) and imaged by confocal and gSTED microscopy. A minimum of 4 ROIs was chosen per cell from representative cell images and used for the analysis. STED images were deconvoluted in Huygens Professional Software (Scientific Volume Imaging) by using the Deconvolution Wizard plug-in, specifying a minimum signal-to-noise ratio of 40 and number of iterations of 100. After threshold adjustment, the image processing package Object Analyzer was used to determine the AQP4-aggregate size, expressed as cluster surface $\left(\mathrm{nm}^{2}\right)$, while the density of AQP4-clusters is expressed as the number of aggregates normalized to the cell surface area. 4-5 cells were analyzed for each independent experiment and condition. Three independent experiments were conducted.

Tissue morphology and Immunohistochemistry. Brain samples were fixed in $10 \%$ of neutral buffered formalin, dehydrated using a graded ethanol series and then embedded in paraffin wax. Serial sections $5 \mu \mathrm{m}$ thick were cut. Rehydrated sections were stained using Mallory's trichrome method for distinguishing cellular from extracellular components. Collagen fibers stained an intense blue, cytoplasm stained reddish, and nucleoli stained pinkish. Images were captured in bright light using an Eclipse E600 photomicroscope and a DMX1200 digital camera (Nikon Instruments SpA, Calenzano, Italy). Pictures of OAP-null and WT brain and cerebellum were taken, under different magnifications to obtain the same fields, in order to assess morphological alterations between WT and OAP-null tissue.

The sections were rehydrated in graded ethanol for immunohistochemistry. Endogenous peroxidases were blocked by incubating sections in PBS containing $1 \% \mathrm{H}_{2} \mathrm{O}_{2}$ for 30 min at RT. After several washes in PBS, the sections were treated with the blocking buffer $1 \%$ normal goat 
serum (Sigma-Aldrich, Saint Louis, MO, USA) in PBS, for $30 \mathrm{~min}$ at RT. After several washes in PBS, sections were incubated in blocking buffer $\mathrm{O} / \mathrm{N}$ at $4{ }^{\circ} \mathrm{C}$ with anti-AQP4 antibody (Santa Cruz Biotechnology, Dallas, TX, USA, goat polyclonal IgG). After several rinses in PBS, sections were incubated with the secondary antibody goat anti-rabbit HRP-conjugate for $1 \mathrm{~h}$ at RT. After several washes in PBS, the immunolabeling was visualized by incubation with 3-30-diaminobenzidine$\mathrm{H}_{2} \mathrm{O}_{2}$ medium for $10 \mathrm{~min}$ at RT. Finally, two sections per slide were counterstained with Hematoxylin and then dehydrated, cleared, and mounted with Entellan. Negative controls were performed by omitting the primary antibodies.

Perfusion and tissue processing for electron microscopy. Mice were deeply anesthetized with an intraperitoneal injection of ketamine $(100 \mathrm{mg} / \mathrm{kg}$ body weight $)$ and transcardially perfused with a solution of $4 \%$ formaldehyde and $0.1 \%$ glutaraldehyde in $0.1 \mathrm{M}$ Phosphate Buffer (PB). The brains were dissected out, post-fixed $\mathrm{O} / \mathrm{N}$ in the fixation solution and stored in a 1:10 dilution of the same solution. Tissue blocks of 1 x $1 \mathrm{~mm}$ from parietal cortex and cerebellum were subjected to the postembedding procedure as described previously (Lunde et al 2015). Briefly, the tissue blocks were cryoprotected in a graded glycerol solution $(10 \%, 20 \%$ and $30 \%$ glycerol in $0.1 \mathrm{M} \mathrm{PB})$, rapidly frozen in liquid propane $\left(-170{ }^{\circ} \mathrm{C}\right)$ and subjected to freeze substitution. Methacrylate resin (Lowicryl HM20) was used to embed the specimens and subjected to UV light for polymerization below $0{ }^{\circ} \mathrm{C}$. Ultrathin sections of $80-100 \mathrm{~nm}$ were cut using an ultratome (Reichert Ultracut $\mathrm{S}$, Leica, Wetzlar, Germany) and the sections were placed on 300 mesh grids.

Immunogold electron microscopy. Immunogold labeling was performed as previously described (Lunde et al., 2015; Hoddevik et al., 2017). Briefly, the sections were incubated O/N with primary antibody, followed by incubation with secondary antibody coupled to $15 \mathrm{~nm}$ colloidal gold particles 
for $90 \mathrm{~min}$. The sections were contrasted with $2 \%$ uranyl acetate and $0.3 \%$ lead citrate. The sections were examined and electron micrographs were acquired using a transmission electron microscope (TEM; Tecnai 12, FEI Company, Eindhoven, Netherlands) at $80 \mathrm{kV}$.

Quantitative PCR. RNA extraction was carried out using Trizol Reagent (Invitrogen, Milan, Italy) according to the instruction manual. Total RNA was quantified by Nanodrop (Thermo, Waltham, MA, USA). $1 \mu \mathrm{g}$ of total RNA was retro transcribed by SuperScript III Reverse Transcriptase (Invitrogen, Milan, Italy). AQP4 total expression was measured by the GAPDH normalized DDCt quantification method using the following primers targeting AQP4 exon 1 (AQP4 For: CGGTTCATGGAAACCTCACC; AQP4 Rev: CATGCTGGCTCCAGTATAAT; GAPDH For: TGCACCACCAACTGCTTAGC; GAPDH Rev: GGCATGGACTGTGGTCATGA). Power up Sybr Green (Invitrogen, Milan, Italy) and StepOne (Applied Biosystems, Thermo, Waltham, MA, USA) were used. All reactions were run in triplicate. After statistical analysis, the data from the different experiments were plotted and averaged in the same bar graph.

Water transport measurements. Volume changes were measured using the validated Calcein fluorescence quenching approach (Hamann et al., 2002; Solenov et al. 2004) using the Flex Station3 plate reader (http://www.moleculardevices.com) equipped with an automatic liquid handling module able to analyze real time fluorescence kinetic data in the 96-well format (Fenton et al. 2010) (Mola et al., 2009, 2016;) . Astrocytes were seeded at a density of 20,000 cells per well and loaded with $10 \mu \mathrm{M}$ of Calcein-AM $24 \mathrm{~h}$ after plating at which time they were $80-85 \%$ confluent. Cells were rinsed in isotonic PBS and hypertonicity was applied $15 \mathrm{~s}$ after the beginning of data acquisition by adding an appropriate volume of hypertonic (D-mannitol $0.5 \mathrm{M}$ ) solution in order to obtain $360 \mathrm{mOsm} / \mathrm{L}$ final extracellular osmolarity. The changes in fluorescence intensity were directly proportional to changes in cell volume. The signal decreases upon addition of hypertonic 
solution as a consequence of water efflux and cell shrinkage. Data acquisition was performed using SoftMax Pro software v5.3, and the data were analyzed with Prism (Graph Pad, San Diego, CA, USA) software. The osmotic properties of the glial cell plasma membranes were analyzed by comparing the time constant obtained by fitting the data with an exponential function.

Experimental design and statistical analysis. All data represent replicates from independent experiments whose number is provided in the figure legends. Statistical analysis was performed in RStudio and GraphPad4 software. First, a Shapiro-Wilk normality test was performed on the data, then statistical significance was evaluated using the unpaired Mann-Whitney test for non-normally distributed data, while the unpaired Student's $t$-test or ANOVA with multiple statistical comparisons between groups (Newman-Keuls test) were performed for normally-distributed data. $P$-values are defined as follows: $* P<0.05, * * P<0.01$, and $* * * P<0.001$. 


\section{RESULTS}

\section{OAP-null mouse model generated by CRISPR/Cas9}

The CRISPR/Cas9 genome editing approach was used to introduce the point mutation (ATG $\rightarrow$ ATC) in a C57BL/6 mouse model in order to obtain a knock-in (KI) mouse having a M23I substitution in the Aqp4 locus at the coding sequence of exon 1 (Ma et al., 1997) (Fig1). Given the concerns about off-target genes potentially targeted by the use of short gRNAs, we decided to generate two different strains bringing the same amino acid substitution of methionine 23 (ATG) to isoleucine (ATC), using two gRNAs (gRNA1 and gRNA2) targeting different AQP4 sequences (Fig 1A). As the $\mathrm{G} \rightarrow \mathrm{C}$ mutation matches the last position for gRNA1, gRNA1 was co-injected with an oligo donor containing an additional silent mutation at the PAM sequence (TCC $\rightarrow$ TCA) to prevent the binding and re-cutting of the sequence by the same gRNA1 after homology-directed repair. In contrast, as the $\mathrm{G} \rightarrow \mathrm{C}$ mutation matches approximately in the middle for gRNA2, gRNA2 was coinjected with an oligo donor not containing any mutation at the PAM sequence (Fig 1B). After the heterozygous were obtained for both strains, the desired mutations in Aqp4 gene were confirmed by DNA sequencing (Fig1C), and heterozygous mice were bred to obtain homozygous offspring. The analysis of live offspring showed a genotype distribution that did not differ significantly from the Mendelian ratio, indicating the absence of pre- or perinatal lethality. Moreover, the animal growth was normal and no sign of suffering phenotype was revealed, as for AQP4 KO mice totally lacking the AQP4 gene (Ma et al., 1997; Fan et al., 2005; Haj-Yasein et al., 2011).

M23I mutation does not affect AQP4 mRNA level and impairs OAP assembly in brain and cerebellum of both strains of OAP-null mice 
The validation and characterization of the two strains of OAP-null mice (indicated as OAP-null.1 and OAP-null.2) was initially performed by qPCR, BN-PAGE and 2D BN/SDS-PAGEImmunoblotting (Fig 2). The first analysis was aimed at verifying whether AQP4 mRNA expression levels were unchanged in OAP-null mice despite the point mutation in the AQP4 gene. The analysis of the total level of AQP4 mRNA performed in the brain and cerebellum of OAP-null and WT mice indicated that M23I mutation did not affect AQP4 gene transcription, leaving AQP4 mRNA levels unaltered compared to WT (Fig 2A).

The presence of multiple AQP4 assemblies, ascribable to AQP4-OAPs of different size (AmiryMoghaddam et al., 2004; Nicchia et al., 2004; Crane et al., 2009), was then analyzed in OAP-null.1 and OAP-null.2 by BN-PAGE followed by immunoblotting using the commercially available antibodies against AQP4 C-terminus (AQP4 Ab) (Fig 2B). The results show that while in all WT tissues several separate bands corresponding to OAPs of different sizes were visible as expected, only a single band corresponding to the smallest AQP4 pool (AQP4 tetramers) was detected in both strains of OAP-null mouse tissues. Densitometric analysis selectively performed on AQP4 tetramers demonstrated an up-regulation in OAP-null mice compared to WT quantified at more than three times higher in the brain and more than twice as high in the cerebellum. The composition of the single AQP4 lower molecular weight pool (AQP4 tetramer) in both strains of OAP-null mice was later analyzed by two-dimensional BN/SDS-PAGE (Fig 2C) followed by immunoblotting performed with AQP4 Ab. Membrane proteins were separated on a first dimension performed under native conditions (BN-PAGE) and a second dimension under denaturing conditions (SDS-PAGE). AQP4 immunoblotting showed several distinct spots containing both M1-AQP4 and M23-AQP4 isoforms with variable expression levels in WT mice. M23-AQP4 was predominant in the largest pool with its expression showing a progressive reduction in the smaller pools. Concomitantly, the M1-AQP4/M23-AQP4 ratio (32 kDa/30 kDa) increased progressively, reaching its maximal level in the smallest pool (arrows), as previously reported (Nicchia et al., 2008). Results obtained in both 
OAP-null mouse strains, in line with the BN/PAGE, showed the selective presence of an upregulated smallest pool and the complete depletion of AQP4 assemblies. The smallest pool, likely corresponding to AQP4 tetramer, was made of both M1-AQP4 and M23-AQP4 (32-30 kDa) in WT mice while it was exclusively made of M1-AQP4 $(32 \mathrm{kDa})$ in both strains of OAP-null mice. The separation of M1-AQP4 and M1-AQP4ex could be generally visualized in immunoblot with a good detection efficiency. No detectable AQP4 protein was revealed in parallel by immunoblot analysis of tissues from AQP4 KO mice.

Western blot experiments performed with AQP4 Ab on brain and cerebellum lysates revealed that both strains of OAP-null mice showed the expected selective loss of the $30 \mathrm{KDa}$ band corresponding to the OAP forming isoform M23-AQP4 (Fig 3A). The AQP4 signal at $32 \mathrm{kDa}$, corresponding to the M1-AQP4 isoform also appeared strongly reduced. The ten times higher OAPnull brain and cerebellum protein sample allowed the correct visualization of M1-AQP4 isoform expression together with the expression of the M1-AQP4ex isoform and a protein band slightly smaller than that of the $30 \mathrm{kDa}$, most likely corresponding to the intracellular AQP4f. An unclear separation between the $32 \mathrm{kDa}(\mathrm{M} 1-\mathrm{AQP} 4)$ and the $30 \mathrm{kDa}$ (M23-AQP4) isoforms usually impaired M1-AQP4 quantitative analysis in WT tissues. With the aim of correctly quantifying M1-AQP4 reduction in OAP-null mice, we generated custom-made antibodies against the N-terminus of M1AQP4 (M1Ab) therefore able to recognize the M1-AQP4 specific sequence selectively. Quantitative analysis of the Western blotting experiments performed with M1 Ab showed that the reduction in M1-AQP4 was approximately $85 \%$ in the brain and 90\% in the cerebellum (Fig. 3 A). These results indicate that in both OAP-null mouse strains the deletion of M23-AQP4 also impairs the amount of M1-AQP4, likely to be that devoted to the OAPs under normal conditions (Fig 3B).

\section{M1-AQP4 based tetramers form functional water channels in brain astrocytes of OAP-null mice}


Astrocyte primary cultures from OAP-null mice were used to assess whether M1-AQP4, in the absence of its molecular partner M23-AQP4, could be normally expressed and form tetramers in the plasma membrane, in a condition in which AQP4 polarization is lost (Nicchia et al., 2000; Solenov et al., 2004). OAP-null astrocyte primary cultures were analyzed for AQP4 assemblies, for M1AQP4 and M23-AQP4 expression and then were used to assess the functionality of AQP4 tetramers by water transport assay (Fig 4). BN-PAGE-Immunoblotting experiments (Fig 4A) showed that AQP4 tetramers were approximately three times as upregulated in OAP-null compared with WT astrocytes. Western blot experiments performed with AQP4 Ab confirmed that in astrocytes from OAP-null mice no detectable M23-AQP4 protein was found while those performed with M1Ab revealed that M1-AQP4 isoform expression in OAP-null mice was about 30\% of the expression level observed in WT mice (Fig 4B).

Fluorescence quenching-based assay was used to compare osmotic volume change rate between OAP-null astrocytes and WT (Fig 4C). The kinetics of cell shrinkage in response to hypertonic challenge was slower in OAP-null $(\tau=4.9 \pm 0.03 \mathrm{~s})$ compared to WT astrocytes $(\tau=3.9 \pm 0.4 \mathrm{~s})$ but faster than those acquired in AQP4 KO astrocytes $(\tau=6.2 \pm 0.22 \mathrm{~s})$. The reduced cell volume shrinkage kinetics in OAP-null astrocytes was in line with the absence of the large majority of AQP4. In contrast, no significant difference was found between WT, OAP-null and AQP4 KO astrocytes in terms of maximal amplitude of cell shrinkage during hypertonic stimulus, in line with previous data (Solenov et al., 2004; Mola et al., 2016). Water transport measurements therefore indicate that M1-AQP4 based tetramers are functionally expressed in the plasma membrane of OAP-null astrocyte primary cultures.

\section{M1-AQP4 based tetramers form smaller aggregates in brain astrocytes of OAP-null mice}

Immunofluorescence experiments shown in Fig 5 were performed to investigate differences in 
AQP4 aggregate size between WT and OAP-null astrocytes by the use of gSTED microscopy. The analysis of AQP4 clusters was performed using AQP4 Ab able to recognize all AQP4 isoforms included AQP4 extended isoforms and the intracellular isoforms. By confocal microscopy analysis, WT astrocytes showed the typical AQP4 plasma membrane pattern and no detectable staining was found in astrocytes from AQP4 KO mice. Astrocytes from OAP-null mice showed a generally weaker AQP4 signal than WT astrocytes (Fig 5A). The size and the distribution analysis of AQP4 assemblies at nanoscale level determined by gSTED microscopy showed a different distribution of AQP4 clusters between WT and OAP-null astrocytes (Fig 5 B, C). In particular, significant differences in AQP4-aggregate median size between genotypes were revealed, compatible with the reduction in the size of AQP4 assembly expected for M1-AQP4 tetramers in the absence of M23AQP4. In line with Western blot results, the density of AQP4 aggregates was significantly reduced in OAP-null astrocytes compared with WT (Fig 5 D).

\section{Limited AQP4 tetramer expression in brain and cerebellum astrocytes}

The massive reduction in AQP4 total expression level, although associated with AQP4 tetramer upregulation in OAP-null mice compared with the WT, induced us to perform immunolocalization experiments aimed at looking for potential specific sites for AQP4 tetramer expression in the brain and cerebellum. Meanwhile a morphological analysis of tissues was performed using histology (Fig 6). From both immunofluorescence and immunocytochemistry, AQP4 tetramers were absent at the perivascular endfeet of the brain cortex where the majority of AQP4 is normally expressed, indicating that M23-AQP4 is here required for the perivascular expression of both M23-AQP4 and M1-AQP4. A very faint and diffuse staining was detectable in OAP-null cerebellum by both immunofluorescence and immunocytochemistry. Morphological analysis revealed no histological differences were found between WT and OAP-null brain and cerebellum. 
Ultrastructure analysis was therefore performed to better ascertain potential specific localization for M1-AQP4 based tetramers. Electron microscopic immunogold investigation of the neocortex (Fig 7), and cerebellum (Fig 8) showed only a few residual immunogold particles in the astrocytic endfeet processes abutting the vessels and pial surface in the area analyzed. We also observed a reduction in the AQP4 immunogold labeling in the astrocytic processes in the neuropil. Immunolocalization studies therefore indicate the absence of preferential AQP4 tetramer expression in the area analyzed. 


\section{DISCUSSION}

The extraordinarily high level of expression of AQP4 water channels at the interfaces between CNS and either blood or cerebrospinal fluid is not unexpected given the important fluid movement in and out of the CNS associated with neuronal activity. However, the need for AQP4 to form quite large supramolecular aggregate OAPs in the plasma membrane is a question still unresolved.

In this study, we have generated a novel mouse model selectively expressing AQP4 under its tetrameric form by introducing a point mutation (M23I) able to abolish the translation of the major OAP forming isoform M23-AQP4, leaving unaltered the possibility to produce the major tetramer forming M1-AQP4 isoform. The aim was to obtain information on the role of M23-AQP4 assembly into OAPs and to investigate whether AQP4 tetramers play a role in CNS astrocytes and can be considered as an alternative to AQP4-OAPs.

Before generating the transgenic mouse, the point mutation able to impair the synthesis of M23AQP4 (M23I), while leaving unaltered the synthesis of M1-AQP4, was characterized in HeLa cells. M1-AQP4 carrying the M23I mutation in transfected cells was shown to be expressed at the same level as wild-type M1-AQP4, to be correctly targeted to the plasma membrane and to give rise to functional AQP4 tetramers unable to aggregate into OAPs (Rossi et al. 2010). Results obtained in cell cultures gave us the rationale to generate the OAP-null mouse model using the same genome design approach. Besides the deletion of the major M23-AQP4 isoform, M23I point mutation was predicted to impair the expression of the minor M23-AQP4ex and the intracellular AQP4d isoforms. Consequently, this model was predicted to selectively express the M1-AQP4 isoform together with M1-AQP4ex, and the expected intracellular spliced variants (AQP4b and AQP4f). The two strains of OAP-null mice gave identical results in terms of phenotype, indicating their specificity for the M23I mutation. 
The first information provided by the biochemical analysis of OAP-null brain astrocytes is that in the absence of M23-AQP4, AQP4 tetramer expression increases whereas the total amount of M1AQP4 is significantly reduced with a massive reduction in the total AQP4. Considering that AQP4 transcription was unaltered in OAP-null mice, a reasonable interpretation of these results could be that reduction in the OAPs is the consequence of the missing M23-AQP4, which additionally results in extra "free" M1-AQP4 forming tetramers and that the majority of M1-AQP4 normally devoted to being part of the OAP assembly, is that which is lost in OAP-null mice, as described in Fig 3B. We can therefore speculate that the large majority of M1-AQP4 in the WT CNS is normally aggregated with M23-AQP4 while the amount of AQP4 tetramers is a minor pool of AQP4. Given that the upregulation of AQP4 tetramers in OAP-null mice is approximately twice that of the WT (2X) and also includes the intracellular AQP4 isoforms which do not form OAPs and that the downregulation of M1-AQP4 protein is approximately eight times higher (8X), we can approximately assume that less than $1 / 16(<6 \%)$ of the total of M1-AQP4 protein is devoted to forming tetramers in the WT while the large majority ( $>94 \%$ ) is part of the OAPs, at least in mouse and human. In rat, and possibly other animals, this proportion could be different due to the expression of AQP4e (Lisjak et al., 2017). It has to be highlighted, however, that an indirect role of intracellular AQP4b and AQP4d isoforms in OAP dynamics has been recently indicated (Lisjak et al., 2020). From the point of view of the molecular physiology of AQP4, this suggests that the assembly of AQP4 into OAPs is a key feature characterizing the molecular identity of this protein channel while the amount of AQP4 tetramers is reduced compared to OAPs under normal conditions. These data also indicate that OAP aggregation is fundamental to secure astrocytes a normal level of AQP4 expression in the plasma membrane and therefore AQP4 tetramers cannot be used by astrocytes as an alternative to OAPs without affecting AQP4 expression levels. This is important in the CNS under physiological and pathophysiological conditions in which OAP aggregation/disaggregation dynamics are implicated, such as astrocyte migration and polarization, brain tumors and Neuromyelitis Optica. 
We here show that although affected by the deletion of M23-AQP4, M1-AQP4 based tetramers can be expressed as functional water channels in astrocytes. This was not unexpected given the large number of studies showing that when transfected separately, M1-AQP4 and M23-AQP4 can form functional water channels (Jung et al., 1994; Furman et al., 2003; Jin et al., 2011; Smith et al., 2014) with a comparable water permeability coefficient (Silberstein et al., 2004).

Light and electron microscopic analysis confirmed the importance of OAPs for AQP4 expression, corroborating the indication that transition from OAPs to AQP4 tetramers would not ensure astrocytes the same level of AQP4 expression. The limited possibilities for M1-AQP4 based tetramers to be expressed in the plasma membrane in the absence of their molecular partner M23AQP4 support the model proposed by Smith and Verkman (2015) that M23-AQP4 and OAPs participate in macromolecular complex formation whereas isolated M1-AQP4 tetramers do not interact strongly with other proteins. We suggest that M23-AQP4 is required for proper M1-AQP4 localization within the OAPs by stabilizing M1-AQP4. This proposed "scaffolding role" of M23AQP4 for M1-AQP4 is consistent with the known strong hydrophobic interactions between the two isoforms (Crane et al., 2009). Notably, the protein scaffolding role has a particular importance for the polarized expression of protein assemblies or complexes in perivascular domains. For instance, alpha syntrophin is considered an important molecular scaffold for the correct assembly of several components of the dystrophin associated protein complex in the perivascular astrocyte domain, including AQP4 (Bragg et al., 2006). We can speculate a sort of domino effect in the scaffolding role of alpha syntrophin on M23-AQP4 in turn acting on M1-AQP4.

Two main functional roles have been ascribed to AQP4 aggregation in OAPs in brain physiology also in the light of previous FFEM data reporting the perivascular microdomains to be characterized by the largest OAPs (Furman et al., 2003). The first is to make AQP4 almost immobile, an 
important property to sustain AQP4 polarized expression at glial endfoot microdomains not delimitated by tight junctions. The second is the substantially high water permeability associated with the packed expression of AQP4 water channels at astrocyte endfeet (Yang and Verkman, 1997). The use of primary astrocyte cultures prepared from the OAP-null mice helped us to answer the question as to whether or not the importance of OAP aggregation is directly associated to AQP4 polarized expression (Smith and Verkman, 2015). It has in fact been largely demonstrated that when astrocytes are placed in culture the polarized expression of AQP4 is completely lost (Nicchia et al., 2000; Solenov et al., 2004). The results obtained in astrocyte primary cultures show that the deletion of M23-AQP4 determines a similar trend, as found in brain and cerebellum, with an upregulation of AQP4 tetramers and a significant reduction in M1-AQP4. This indicates that AQP4 expression under its tetrameric form in astrocyte plasma membrane is massively reduced compared to the possibility for AQP4 expression under the OAP form, even in the absence of AQP4 polarized expression. However, based on the higher AQP4 tetramer upregulation and the lower M1-AQP4 downregulation in OAP-null primary cultured astrocytes compared to OAP-null brain and cerebellum, it is likely that the amount of M1-AQP4 devoted to the OAPs is lower in astrocyte cultures (non polarized AQP4 expression) compared with brain and cerebellum astrocytes (AQP4 polarized expression). This indicates that it is likely that an additional need for AQP4 aggregation is associated with AQP4 polarized expression.

Besides the CNS, AQP4 is expressed in various organs such as the skeletal muscle (Frigeri et al. 1995b) kidney (Frigeri et al. 1995a), stomach (Frigeri et al. 1995b) and lung (Frigeri et al. 1995a) . The preliminary analysis of M1-AQP4 expression and AQP4 supramolecular organization in the kidney of OAP-null mice (Figure 4 Supplementary Information) has revealed an opposite trend with a downregulation of AQP4 tetramers associated with a massive reduction in M1-AQP4 expression which is undetectable by Western blot. This data indicates that the role of AQP4 isoforms into OAP assembly dynamics might depend on the cell type and that this mouse model could be useful to 
clarify these aspects.

AQP4-null mice totally lacking the AQP4 gene have largely been used to assess whether AQP4 plays a key role in organ physiology (Haj-Yasein et al. 2011; Ma et al. 1997; Verkman et al. 2006). However, though AQP4 deletion is associated with a number of functional defects, such as impaired hearing and olfaction ( $\mathrm{Li}$ and Verkman 2001), edema (Papadopoulos et al. 2004), epilepsy (Binder et al. 2004), stroke (Manley et al. 2000) and a mild concentrating defect in the kidney (Verkman et al. 2000), from the analysis of these phenotype studies it has emerged that deletion of AQP4 is not associated with any apparent phenotypic abnormality (Verkman et al. 2000). In this study, we have assessed that the OAP-null mouse growth is normal and no sign of suffering phenotype is revealed, in line with AQP4 KO mice totally lacking the AQP4 gene (Fan et al. 2005; Haj-Yasein et al. 2011; Ma et al. 1997). Moreover, no gross brain morphological abnormalities have been detected in line with previous data (Saadoun et al. 2009). However, future studies would need to be performed to ascertain whether and how the possibility to express the freely mobile M1AQP4 in OAP-null mice can affect glial cell migration/invasiveness, adhesion, polarization and apoptosis as is predictable based on the current literature (Simone et al. 2019; Smith et al. 2014).

To the best of our knowledge, this is the first study in which the concern over off-target cleavage in CRISPR-mediated genome editing has been considered important for potential corresponding offtarget biological effects in animal models. The entirely comparable results obtained between the two strains of OAP-null mice in terms of genotype-phenotype correlation give robustness to the main findings here shown and provide support to the affordability of CRISPR/Cas9 recently debated (Zhang et al., 2015; Schaefer et al., 2017; Iyer et al., 2018; Montoliu and Whitelaw, 2018).

In conclusion, this study demonstrates for the first time in vivo that the OAP supramolecular structure is a key feature characterizing the molecular identity of the AQP4 water channel and that 
the large majority of AQP4 is organized into OAPs under physiological condition, even independently of AQP4 polarized expression at astrocyte endfeet. The key information emerging from the characterization of the OAP-null mouse model here is also that OAP aggregation is essential to guarantee astrocytes a normal level of AQP4 expression in the plasma membrane and therefore AQP4 tetramers cannot be used by astrocytes as an alternative to OAPs without affecting AQP4 total expression levels. This is important in the light of astrocyte physiological and pathophysiological conditions in which OAP aggregation/disaggregation dynamics have been implicated, such as astrocyte migration and polarization, brain tumors and Neuromyelitis Optica. In particular, transitions from large OAPs to smaller OAPs or tetramers have been proposed to be regulated depending on cell physiological need (astrocyte migration or differentiation) without altering astrocyte permeability (Smith and Verkman, 2015). OAP dynamics can also influence the fate of glioma cells (Simone et al., 2019) toward cell migration or apoptosis and AQP4 aggregation into OAPs has a key role in Neuromyelitis Optica where NMO IgG autoantibodies can bind their autoantigen AQP4 only when aggregated into OAPs (Nicchia et al., 2009; Crane et al., 2011; Pisani et al., 2011). The OAP-null model could be useful for future studies aimed at identifying mechanisms underlying OAP biology and understanding the role of AQP4 aggregation dynamics under the physiological and pathophysiological circumstances mentioned above. It would also be a suitable model for assessing the role of the new emerging AQP4 intracellular and C-terminus extended AQP4 isoforms in cell biology and in OAP formation as well as for analyzing whether the alteration of AQP4 aggregation properties can be the basis of impaired organ function in those tissues in which AQP4 is expressed. We believe this study provides important clues to elucidate the cellular and molecular mechanism underpinning the role of astrocyte health and disease. 


\section{REFERENCES}

Amiry-Moghaddam M, Frydenlund DS, Ottersen OP. 2004a. Anchoring of aquaporin-4 in brain: molecular mechanisms and implications for the physiology and pathophysiology of water transport. Neuroscience 129(4):999-1010.

Amiry-Moghaddam M, Xue R, Haug FM, Neely JD, Bhardwaj A, Agre P, Adams ME, Froehner SC, Mori S, Ottersen OP. 2004b. Alpha-syntrophin deletion removes the perivascular but not endothelial pool of aquaporin-4 at the blood-brain barrier and delays the development of brain edema in an experimental model of acute hyponatremia. Faseb J 18(3):542-4.Badaut J, Fukuda AM, Jullienne A, Petry KG. 2014. Aquaporin and brain diseases. Biochim Biophys Acta - Gen Subj 1840:1554-1565.

Badaut J, Fukuda AM, Jullienne A, Petry KG. 2014. Aquaporin and brain diseases. Biochim Biophys Acta 1840(5):1554-65.

Benfenati V, Caprini M, Dovizio M, Mylonakou MN, Ferroni S, Ottersen OP, Amiry-Moghaddam M. 2011. An aquaporin-4/transient receptor potential vanilloid 4 (AQP4/TRPV4) complex is essential for cell-volume control in astrocytes. Proc Natl Acad Sci U S A 108:2563-2568.

Binder DK, Oshio K, Ma T, Verkman AS, Manley GT. 2004. Increased seizure threshold in mice lacking aquaporin-4 water channels. Neuroreport 15(2):259-62.

Bragg AD, Amiry-Moghaddam M, Ottersen OP, Adams ME, Froehner SC. 2006. Assembly of a perivascular astrocyte protein scaffold at the mammalian blood-brain barrier is dependent on $\alpha$-syntrophin. Glia 53:879-890.

Crane JM, Bennett JL, Verkman AS. 2009. Live cell analysis of aquaporin-4 M1/M23 interactions and regulated orthogonal array assembly in glial cells. J Biol Chem 284:35850-35860.

Crane JM, Lam C, Rossi A, Gupta T, Bennett JL, Verkman AS. 2011. Binding affinity and specificity of neuromyelitis optica autoantibodies to aquaporin-4 M1/M23 isoforms and 
orthogonal arrays. J Biol Chem 286:16516-16524.

De Bellis M, Pisani F, Mola MG, Rosito S, Simone L, Buccoliero C, Trojano M, Nicchia GP, Svelto M, Frigeri A. 2017. Translational readthrough generates new astrocyte AQP4 isoforms that modulate supramolecular clustering, glial endfeet localization, and water transport. Glia 65:790-803.

Fan Y, Zhang J, Sun XL, Gao L, Zeng XN, Ding JH, Cao C, Niu L, Hu G. 2005. Sex- and regionspecific alterations of basal amino acid and monoamine metabolism in the brain of aquaporin-4 knockout mice. J Neurosci Res 82:458-464.

Fenton RA, Moeller HB, Nielsen S, de Groot BL, Rutzler M. 2010. A plate reader-based method for cell water permeability measurement. Am J Physiol Renal Physiol 298(1):F224-30.

Frigeri A, Gropper MA, Turck CW, Verkman AS. 1995a. Immunolocalization of the mercurialinsensitive water channel and glycerol intrinsic protein in epithelial cell plasma membranes. Proc Natl Acad Sci U S A 92(10):4328-31.

Frigeri A, Gropper MA, Umenishi F, Kawashima M, Brown D, Verkman AS. 1995b. Localization of MIWC and GLIP water channel homologs in neuromuscular, epithelial and glandular tissues. J Cell Sci 108 ( Pt 9):2993-3002.

Furman CS, Gorelick-Feldman DA, Davidson KGV, Yasumura T, Neely JD, Agre P, Rash JE. 2003. Aquaporin-4 square array assembly: Opposing actions of M1 and M23 isoforms. Proc Natl Acad Sci U S A 100:13609-13614.

Haj-Yasein NN, Vindedal GF, Eilert-Olsen M, Gundersen GA, Skare Ø, Laake P, Klungland A, Thorén AE, Burkhardt JM, Ottersen OP, Nagelhus EA. 2011. Glial-conditional deletion of aquaporin-4 (Aqp4) reduces blood-brain water uptake and confers barrier function on perivascular astrocyte endfeet. Proc Natl Acad Sci U S A 108:17815-17820.

Hamann S, Kiilgaard JF, Litman T, Alvarez-Leefmans FJ, Winther BR, Zeuthen T. 2002. 
Measurement of cell-volume changes by fluorescence self-quenching. J Fluor 12: 139-145..

Hoddevik EH, Khan FH, Rahmani S, Ottersen OP, Boldt HB, Amiry-Moghaddam M. 2017. Factors determining the density of AQP4 water channel molecules at the brain-blood interface. Brain Struct Funct 222:1753-1766.

Iyer V, Boroviak K, Thomas M, Doe B, Riva L, Ryder E, Adams DJ. 2018. No unexpected CRISPR-Cas9 off-target activity revealed by trio sequencing of gene-edited mice. PLoS Genet 14.

Jin BJ, Rossi A, Verkman AS. 2011. Model of aquaporin-4 supramolecular assembly in orthogonal arrays based on heterotetrameric association of M1-M23 isoforms. Biophys J 100:2936-2945.

Jung JS, Prestont GM, Smith BL, Guggino WB, Agre P. 1994. Molecular structure of the water channel through aquaporin CHIP: The hourglass model. J Biol Chem 269:14648-14654.

Li J, Verkman AS. 2001. Impaired hearing in mice lacking aquaporin-4 water channels. J Biol Chem 276(33):31233-7.

Lisjak M, Potokar M, Rituper B, Jorgacevski J, Zorec R. 2017. AQP4e-Based Orthogonal Arrays Regulate Rapid Cell Volume Changes in Astrocytes. J Neurosci 37(44):10748-10756.

Lisjak M, Potokar M, Zorec R, Jorgačevski J. 2020. Indirect Role of AQP4b and AQP4d Isoforms in Dynamics of Astrocyte Volume and Orthogonal Arrays of Particles. Cells 9(3):735.

Lunde LK, Camassa LMA, Hoddevik EH, Khan FH, Ottersen OP, Boldt HB, Amiry-Moghaddam M. 2015. Postnatal development of the molecular complex underlying astrocyte polarization. Brain Struct Funct 220:2087-2101.

Ma T, Yang B, Gillespie A, Carlson EJ, Epstein CJ, Verkman AS. 1997. Generation and phenotype of a transgenic knockout mouse lacking the mercurial-insensitive water channel aquaporin-4. $\mathrm{J}$ Clin Invest 100:957-962.

Manley GT, Fujimura M, Ma T, Noshita N, Filiz F, Bollen AW, Chan P, Verkman AS. 2000. 
Aquaporin-4 deletion in mice reduces brain edema after acute water intoxication and ischemic stroke. Nat Med 6(2):159-63.

Moe SE, Sorbo JG, Sogaard R, Zeuthen T, Petter Ottersen O, Holen T. 2008. New isoforms of rat Aquaporin-4. Genomics 91(4):367-77.

Mola MG, Nicchia GP, Svelto M, Spray DC, Frigeri A. 2009. Automated Cell-Based Assay for Screening of Aquaporin Inhibitors. Anal Chem 81:8219-8229.

Mola MG, Sparaneo A, Gargano CD, Spray DC, Svelto M, Frigeri A, Scemes E, Nicchia GP. 2016. The speed of swelling kinetics modulates cell volume regulation and calcium signaling in astrocytes: A different point of view on the role of aquaporins. Glia 64:139-54.

Montoliu L, Whitelaw CBA. 2018. Unexpected mutations were expected and unrelated to CRISPRCas9 activity. Transgenic Res 27:315-319.

Nagelhus EA, Ottersen OP. 2013. Physiological Roles of Aquaporin-4 in Brain. Physiol Rev 93:1543-1562.

Neely JD, Christensen BM, Nielsen S, Agre P. 1999. Heterotetrameric composition of aquaporin-4 water channels. Biochemistry 38:11156-11163.

Neuhaus J. 1990. Orthogonal arrays of particles in astroglial cells: Quantitative analysis of their density, size, and correlation with intramembranous particles. Glia 3:241-251.

Nicchia GP, Cogotzi L, Rossi A, Basco D, Brancaccio A, Svelto M, Frigeri A. 2008. Expression of multiple AQP4 pools in the plasma membrane and their association with the dystrophin complex. J Neurochem 105:2156-2165.

Nicchia GP, Frigeri A, Liuzzi GM, Santacroce MP, Nico B, Procino G, Quondamatteo F, Herken R, Roncali L, Svelto M. 2000. Aquaporin-4-containing astrocytes sustain a temperature- and mercury-insensitive swelling in vitro. Glia 31:29-38.

Nicchia GP, Mastrototaro M, Rossi A, Pisani F, Tortorella C, Ruggieri M, Lia A, Trojano M, 
Frigeri A, Svelto M. 2009. Aquaporin-4 orthogonal arrays of particles are the target for neuromyelitis optica autoantibodies. Glia 57:1363-1373.

Nicchia GP, Nico B, Camassa LMA, Mola MG, Loh N, Dermietzel R, Spray DC, Svelto M, Frigeri A. 2004. The role of aquaporin-4 in the blood-brain barrier development and integrity: Studies in animal and cell culture models. Neuroscience 129:935-944.

Nielsen S, Nagelhus EA, Amiry-Moghaddam M, Bourque C, Agre P, Ottersen OR. 1997. Specialized membrane domains for water transport in glial cells: High- resolution immunogold cytochemistry of aquaporin-4 in rat brain. J Neurosci 17:171-180.

Noell S, Ritz R, Wolburg-Buchholz K, Wolburg H, Fallier-Becker P. 2012. An allograft glioma model reveals the dependence of Aquaporin-4 expression on the brain microenvironment. PLoS One 7:2-8.

Palazzo C, Buccoliero C, Mola MG, Abbrescia P, Nicchia GP, Trojano M, Frigeri A. 2019. AQP4ex is crucial for the anchoring of AQP4 at the astrocyte end-feet and for neuromyelitis optica antibody binding. Acta Neuropathol Commun 7:51.

Papadopoulos MC, Manley GT, Krishna S, Verkman AS. 2004. Aquaporin-4 facilitates reabsorption of excess fluid in vasogenic brain edema. Faseb J 18(11):1291-3.

Papadopoulos MC, Verkman AS. 2012. Aquaporin 4 and neuromyelitis optica. Lancet Neurol $11: 535-544$.

Pisani F, Mastrototaro M, Rossi A, Nicchia GP, Tortorella C, Ruggieri M, Trojano M, Frigeri A, Svelto M. 2011. Identification of two major conformational aquaporin-4 epitopes for neuromyelitis optica autoantibody binding. J Biol Chem 286:9216-24.

Rash JE, Davidson KGV, Yasumura T, Furman CS. 2004. Freeze-fracture and immunogold analysis of aquaporin-4 (AQP4) square arrays, with models of AQP4 lattice assembly. Neuroscience 129:915-934. 
Rossi A, Crane JM, Verkman AS. 2011. Aquaporin-4 Mz isoform: brain expression, supramolecular assembly and neuromyelitis optica antibody binding. Glia 59(7):1056-63.

Rossi A, Moritz TJ, Ratelade J, Verkman AS. 2012. Super-resolution imaging of aquaporin-4 orthogonal arrays of particles in cell membranes. J Cell Sci 125:4405-4412.

Rossi A, Pisani F, Nicchia GP, Svelto M, Frigeri A. 2010. Evidences for a Leaky Scanning Mechanism for the Synthesis of the Shorter M23 Protein Isoform of Aquaporin-4: Implication in orthogonal array formation and Neuromyelitis optica antibody interaction. J Biol Chem $285: 4562-4569$.

Saadoun S, Tait MJ, Reza A, Davies DC, Bell BA, Verkman AS, Papadopoulos MC. 2009. AQP4 gene deletion in mice does not alter blood-brain barrier integrity or brain morphology. Neuroscience 161(3):764-72.

Schaägger H, Cramer WA, von Jagow G. 1994. Analysis of molecular masses and oligomeric states of protein complexes by blue native electrophoresis and isolation of membrane protein complexes by two-dimensional native electrophoresis. Anal Biochem 217:220-230.

Schaefer KA, Wu WH, Colgan DF, Tsang SH, Bassuk AG, Mahajan VB. 2017. Unexpected mutations after CRISPR-Cas9 editing in vivo. Nat Methods 14:547-548.

Silberstein C, Bouley R, Huang V, Fang P, Pastor-Soler N, Brown D, Van Hoek AN. 2004. Membrane organization and function of M1 and M23 isoforms of aquaporin-4 in epithelial cells. Am J Physiol - Ren Physiol 287.

Simone L, Pisani F, Mola MG, De Bellis M, Merla G, Micale L, Frigeri A, Vescovi AL, Svelto M, Nicchia GP. 2019. AQP4 aggregation state is a determinant for glioma cell fate. Cancer Res 79:2182-2194.

Smith AJ, Jin B-J, Ratelade J, Verkman AS. 2014. Aggregation state determines the localization and function of M1- and M23-aquaporin-4 in astrocytes. J Cell Biol 204:559-573. 
Smith AJ, Verkman AS. 2015. Superresolution Imaging of Aquaporin-4 Cluster Size in AntibodyStained Paraffin Brain Sections. Biophys J 109:2511-2522.

Solenov E, Watanabe H, Manley GT, Verkman AS. 2004. Sevenfold-reduced osmotic water permeability in primary astrocyte cultures from AQP-4-deficient mice, measured by a fluorescence quenching method. Am J Physiol - Cell Physiol 286.

Verkman AS, Binder DK, Bloch O, Auguste K, Papadopoulos MC. 2006. Three distinct roles of aquaporin-4 in brain function revealed by knockout mice. Biochim Biophys Acta 1758(8):1085-93.

Verkman AS, Ratelade J, Rossi A, Zhang H, Tradtrantip L. 2012. Aquaporin-4: orthogonal array assembly, CNS functions, and role in neuromyelitis optica. Acta Pharmacol Sin 32(6):702-10.

Verkman AS, Yang B, Song Y, Manley GT, Ma T. 2000. Role of water channels in fluid transport studied by phenotype analysis of aquaporin knockout mice. Exp Physiol 85 Spec No:233S$241 \mathrm{~S}$.

Verkman AS, Rossi A, Crane JM. 2012. Live-cell imaging of aquaporin-4 supramolecular assembly and diffusion. In: Methods in Enzymology. Vol. 504. Academic Press Inc. p 341-354.

Wilcock DM, Vitek MP, Colton CA. 2009. Vascular amyloid alters astrocytic water and potassium channels in mouse models and humans with Alzheimer's disease. Neuroscience 159:10551069.

Yang B, Verkman AS. 1997. Water and glycerol permeabilities of aquaporins 1-5 and MIP determined quantitatively by expression of epitope-tagged constructs in Xenopus oocytes. $\mathrm{J}$ Biol Chem 272:16140-6.

Zhang XH, Tee LY, Wang XG, Huang QS, Yang SH. 2015. Off-target effects in CRISPR/Cas9mediated genome engineering. Mol Ther - Nucleic Acids 4:e264. 

GLIA

John Wiley \& Sons, Inc. 


\section{FIGURE LEGENDS}

Figure 1. Generation of OAP-null mouse using the CRISPR/Cas9 system. A) Schematic representation of the targeted exon 1 of mouse AQP4 on chromosome 18. Coding exons are shown as solid black bars and are interspaced by non-coding sequences represented with thin lines. Two overlapping gRNAs, gRNA1 (red line) and gRNA2 (blue line), were used to target different AQP4 sequences of the wildtype allele. The proto spacer-adjacent motif (PAM) sequences are underlined for each gRNA (PAM1 for gRNA1 and PAM2 for gRNA2) while the single base (G) to be mutated is highlighted by an asterisk in the wildtype allele before targeting. The green scissors indicate the Cas9 cleavage site. The mutated base "C" $(\mathrm{G} \rightarrow \mathrm{C})$ is highlighted in red in the mutant alleles obtained after targeting. The mutated allele obtained with gRNA1 displayed an additional silent mutation (in grey) at the PAM sequence. B) The genome of wildtype mice with C57BL/6 genetic background was edited by injecting Cas9 (in green), donor oligo sequences containing desired mutations and gRNAs (gRNA1 in red and gRNA2 in blue) in fertilized eggs. C) Sequence analysis of the mutant alleles in heterozygous mice. The desired mutation $\mathrm{G} \rightarrow \mathrm{C}$ (red arrows) in the $A Q P 4$ gene was confirmed in both gRNA1 and gRNA2 strains of KI mice. The silent mutation $(\mathrm{C} \rightarrow \mathrm{A})$ at the PAM sequence is shown in grey for gRNA1 mutant allele.

Figure 2. Analysis of AQP4 mRNA by qPCR and AQP4 assemblies by BN-PAGE and BN/SDS-PAGE in WT and OAP-null brain and cerebellum. A) Real time PCR analysis of relative AQP4 mRNA levels in the brain and cerebellum from WT and OAP-null mice. AQP4 mRNA levels were significantly higher in the cerebellum compared with the brain but there were no significant differences between tissues from WT and OAP-null mice (unpaired Student's $t$-test: $* * * P<0.001, \mathrm{n}=3$. B) Left: membrane proteins extracted from the brain and cerebellum of OAPnull.1, OAP-null.2 and WT mice were separated under native conditions on 3-9\% acrylamide gels (BN-PAGE) and then subjected to immunoblot using AQP4 antibodies against all AQP4 isoforms 
(AQP4 Ab). Tissues from AQP4 KO mice $(\mathrm{KO})$ were used as negative controls. In WT tissues, AQP4 tetramers (arrow) and several different pools corresponding to different sized AQP4 assemblies (arrowheads) are visualized. In contrast, in OAP-null tissues only the smaller AQP4 pool corresponding to AQP4 tetramers is visualized. Right: Bar graph summarizing the densitometric analysis, shown as arbitrary units (A.U.), performed on AQP4 tetramers indicated by the arrow in the tissues, as indicated. Data from OAP null.1 and OAP-null.2 have been pooled together since no significant difference was found between the two strains (unpaired Student's $t$ test: $\left.{ }^{*} P<0.05,{ }^{*} P<0.01, \mathrm{n}=3-6\right)$. Data in $\left.\mathbf{A}\right)$ and $\left.\mathbf{B}\right)$ are expressed as means \pm standard error of the mean (SEM). C) Two-dimensional analysis of AQP4 assemblies in WT and OAP-null brain and cerebellum. After being separated under native conditions in 3-9\% acrylamide gels (BN-PAGE, first dimension), membrane proteins were subjected to a second dimension performed under denaturing condition (SDS-PAGE), using a $13 \%$ acrylamide gel. Immunoblot was performed using AQP4 Ab. Tissues from AQP4 KO (KO) mice were used as negative controls. AQP4 tetramers are indicated by vertical arrows and AQP4 assemblies by arrowheads. M1-AQP4 (M1) and M1AQP4ex (M1ex) are indicated by horizontal arrows.

Figure 3. Western blot analysis of M1-AQP4 and M23-AQP4 expression in WT and OAP-null brain and cerebellum. A) Left: Western blot analysis of M1-AQP4 (M1) and M23-AQP4 (M23) expression in the brain and cerebellum from WT, OAP-null (OAP-null.1 and OAP-null.2) and AQP4 KO (KO) mice, performed using AQP4 Ab and antibodies able to recognize the M1-AQP4 isoform $(\mathrm{M} 1 \mathrm{Ab})$ selectively. The asterisk indicates a non specific band recognized by M1 Ab in all the brain and cerebellum tissues analyzed, including those from AQP4 KO mice, differing in size between brain and cerebellum. In the Western blot performed with AQP4 Ab, a ten-times (10X) or a 5 times (5X) higher concentration of OAP null tissues was loaded to visualize the correct expression of the M1-AQP4 isoform. In the 5X and 10X samples from OAP-null mice, the M1AQP4ex isoform (M1ex) and a band presumably corresponding to AQP4f (arrowhead) are 
indicated. Right: bar graphs summarizing the densitometric analysis of M1-AQP4 (M1/ Coomassie Blue, shown in \%) quantified from Western blot performed using M1 Ab in brain and cerebellum, as indicated. Coomassie Blue staining of total proteins was used as an internal control for protein loading (unpaired Student's $t$-test: $* * * P<0.001, \mathrm{n}=6$ ). Data are expressed as means $\pm \mathrm{SEM}$. B) Schema showing the effect of M23-AQP4 deletion on its molecular partner M1-AQP4 and on AQP4 tetramer expression based on the data shown in Figure 2B and Figure 3A.

Figure 4. Analysis of AQP4 assemblies in WT and OAP-null astrocyte primary cultures by BN-PAGE, Western blot, and water transport assay. A) Left: Membrane proteins extracted from OAP-null and WT astrocytes were separated under native conditions in 3-9\% acrylamide gels (BNPAGE) and then subjected to immunoblot analysis using AQP4 Ab. Astrocytes from AQP4 KO mice $(\mathrm{KO})$ were used as negative controls. AQP4 tetramers are indicated by an arrow and AQP4 assemblies by arrowheads. Right: bar graph summarizing the densitometric analysis, shown as arbitrary units (A.U.) performed on AQP4 tetramer in WT and OAP-null astrocytes (unpaired Student's $t$-test: $* * P<0.01, \mathrm{n}=3)$. B) Left: Western blot analysis of AQP4 expression in astrocytes from WT, OAP-null and AQP4 KO (KO) mice, as indicated, performed using AQP4 Ab and M1 Ab. Right: bar graph summarizing the densitometric analysis of M1-AQP4 (M1/ Coomassie Blue, shown in \%) quantified from Western blot performed using M1 Ab in WT and OAP-null astrocyte primary cultures. Coomassie Blue staining was used as an internal control for protein loading (unpaired Student's $t$-test: $* * * P<0.001, \mathrm{n}=3$ ). C) Left: Functional analysis for AQP4 mediated water transport performed with the calcein quenching assay using the Flex Station multiplate reader. The typical shrinkage kinetics induced by osmotic shocks (hypertonic solution) and recorded from calcein-AM loaded WT, OAP-null and AQP4 KO (KO) astrocytes is shown. Middle: Bar graph of the mean \pm SEM values of time constants obtained by fitting an exponential curve to the shrinkage of cells after exposure to 360 mOsm solution. Right: Bar graph showing the mean \pm 
SEM values of the maximal amplitude of astrocyte shrinkage (ANOVA followed by NewmanKeuls test: $\left.* P<0.05,{ }^{*} P<<0.01 ; * * * P<0.001, \mathrm{n}=3\right)$.

Figure 5. Analysis of AQP4 assemblies in WT and OAP-null astrocyte primary cultures by gSTED microscopy. A) Left and middle panels: representative images of WT, OAP-null and KO astrocytes, stained with AQP4 Ab (green) and GFAP Ab (red) obtained by confocal microscopy. Right panels: boxed yellow areas are shown at higher magnification with AQP4-aggregates imaged by confocal (upper insets) and gSTED (lower insets) microscopy, as indicated. Scale bars: $10 \mu \mathrm{m}$ (whole-cell); $500 \mathrm{~nm}$ (confocal and STED insets). B) AQP4-aggregate surface $\left(\mathrm{nm}^{2}\right)$ frequency histogram of WT (left) and OAP-null (right) astrocytes, showing the number of analyzed clusters plotted against the cluster dimension. C) Differences in AQP4-aggregate median size between genotypes. In the box plot, the box represents the interquartile range (IQR), the central line is the median, the plus sign ' + ' identifies the mean values, and the whiskers extend from the minimum to the maximum values (Shapiro-Wilk normality test $* * * \quad \mathrm{P}<0.001$ followed by unpaired MannWhitney test: $* * * P<0.001, \mathrm{n}=3$ ). D) Quantification of AQP4-aggregate density expressed as the number of STED-identified clusters normalized to the cell surface area. Data are presented as mean \pm SEM (unpaired Student T-test: $* * * P<0.001, \mathrm{n}=3$ ).

Figure 6. AQP4 distribution and morphological analysis on OAP-null and WT brain and cerebellum. AQP4 localization by immunofluorescence, immunoperoxidase and trichrome stain of brain cortex and cerebellum sections prepared from WT and OAP-null mice. Arrows indicate AQP4 staining which is clearly visible and specific in the WT tissues and very faint and diffuse in OAPnull tissues. CX, cortex; M, molecular cell layer; G, granular cell layer. Scale bar: $100 \mu \mathrm{m}$. 
Figure 7. AQP4 immunogold labeling in OAP-null and WT cortex. Electron micrographs showing AQP4 immunogold labeling in cortex of WT (A and C) and OAP-null (B and D) mice. No AQP4 immnunogold labeling is visible at the perivascular astrocytic (B) membrane and only a few immunogold particles are present at the subpial endfoot (D) of the OAP-null mouse cortex. A and B are from perivascular membranes (arrows), while C and D are from subpial membranes (arrows). L $=$ lumen, $\mathrm{E}=$ endothelium, $\mathrm{P}=$ pericyte, asterisk = basal membrane. Scale bars $(\mathrm{A}, \mathrm{B}: 500 \mathrm{~nm} ; \mathrm{C}$, $\mathrm{D}: 1 \mu \mathrm{m})$.

Figure 8. AQP4 immunogold labeling o in OAP-null and WT cerebellum. Electron micrographs showing AQP4 immunogold labeling in cerebellum of WT (A, C, E) and OAP-null mice (B, D, F). Note the strong reduction in the perivascular and subpial AQP4 immunogold labeling of the OAPnull mice compared to the WT. Immunogold particles indicating AQP4 labeling in astrocyte processes in the white matter of cerebellum are shown with double-headed arrows. A and B are from perivascular membranes (arrows) in the granule layer. C and D are from perivascular membranes (arrows) in the white matter. $\mathrm{E}$ and $\mathrm{F}$ are from subpial membranes (arrows). L = lumen, $\mathrm{E}=$ endothelium, $\mathrm{P}=$ pericyte, $\mathrm{Ax}=$ axon, $\mathrm{my}=$ myelin, doubled arrows $=$ other astrocyte membranes, asterisk $=$ basal membrane, arrowheads $=$ collagen fibers of pial mater. Scale bars $(\mathrm{C}$ : $500 \mathrm{~nm} ; \mathrm{A}, \mathrm{B}, \mathrm{D}-\mathrm{F}: 1 \mu \mathrm{m})$

Figure 4 Supplementary Information. Analysis of AQP4 assemblies and M1-AQP4 and M23AQP4 expression by BN-PAGE, BN/SDS-PAGE and Western blot in WT and OAP-null kidney plasma membrane vesicles. Membrane proteins were extracted from plasma membrane vesicles prepared from the kidney medulla of OAP-null and WT mice as follows: mouse tissue was cut into small pieces and homogenized with a Dounce apparatus in five volumes of ice-cold Homogenizing Buffer (250 mM Sucrose; $10 \mathrm{mM}$ Tris-HCl, $\mathrm{pH}$ 7,5) added with protease inhibitor cocktail. Homogenates were centrifuged at $4,000 \mathrm{xg}$ for 10 minutes at $4^{\circ} \mathrm{C}$. After centrifugation, 
pellets were discarded and supernatants collected and centrifuged at $17,000 \mathrm{xg}$ for 45 minutes at $4^{\circ} \mathrm{C}$ to obtain a low-speed membrane vesicles-enriched pellet. The resulting pellets were resuspended in seven volumes of BN-PAGE lysis buffer added with protease inhibitor for one hour on ice and vortexed every 10 minutes. Vesicle lysates were then centrifuged at $21,000 \mathrm{xg}$ for one hour at $4^{\circ} \mathrm{C}$. The supernatant was collected and the protein content was measured using the $\mathrm{BCA}^{\mathrm{TM}}$ Protein Assay Kit (http://www.thermoscientific.com).

A) Extracted proteins were separated under native conditions on 3-9\% acrylamide gels (BN-PAGE) and then subjected to immunoblot using AQP4 antibodies against all AQP4 isoforms (AQP4 Ab). AQP4 tetramers are indicated by the arrow and pools corresponding to AQP4 assemblies are indicated by arrowheads. Right: Bar graph summarizing the densitometric analysis, shown as arbitrary units (A.U.), performed on AQP4 tetramers indicated by the arrow in the tissues, as indicated (unpaired Student's $t$-test, ${ }^{*} P<0.05, \mathrm{n}=3$ ). Data are expressed as means \pm standard error of the mean (SEM). B) Two-dimensional analysis of AQP4 assemblies in WT and OAP-null kidney plasma membrane vesicles. After being separated under native conditions in 3-9\% acrylamide gels (BN-PAGE, first dimension), membrane proteins were subjected to a second dimension performed under denaturing condition (SDS-PAGE), using a $13 \%$ acrylamide gel. Immunoblot was performed using AQP4 Ab. AQP4 tetramers are indicated by vertical arrows and AQP4 assemblies by arrowheads. C) Western blot analysis of M1-AQP4 (M1) and M23-AQP4 (M23) expression in the vesicles from WT and OAP-null mice, performed using AQP4 Ab. Brain tissues were used as control. A seven-times (7X) or three-times (3X) higher concentration of OAP null tissues was loaded to visualize the expression of the M1-AQP4 isoform.

The results show that no tetramer upregulation occurs in OAP-null kidneys and that M1-AQP4 expression is massively reduced and undetectable by Western blot even using a seven-times higher concentration of protein sample compared with the WT. 
A

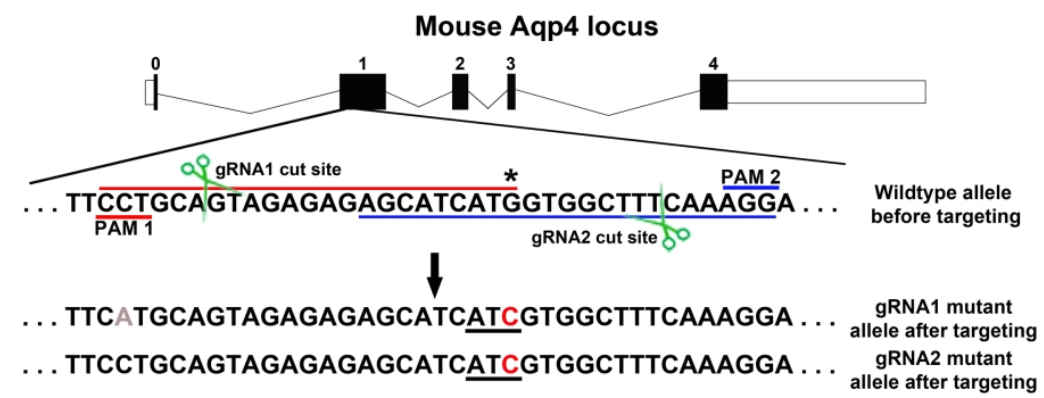

B Donor oligo sequence
with gRNA1 .. TTCATGCAGTAGAGAGAGCATCATCGTGGCTTTCAAAGGA ...

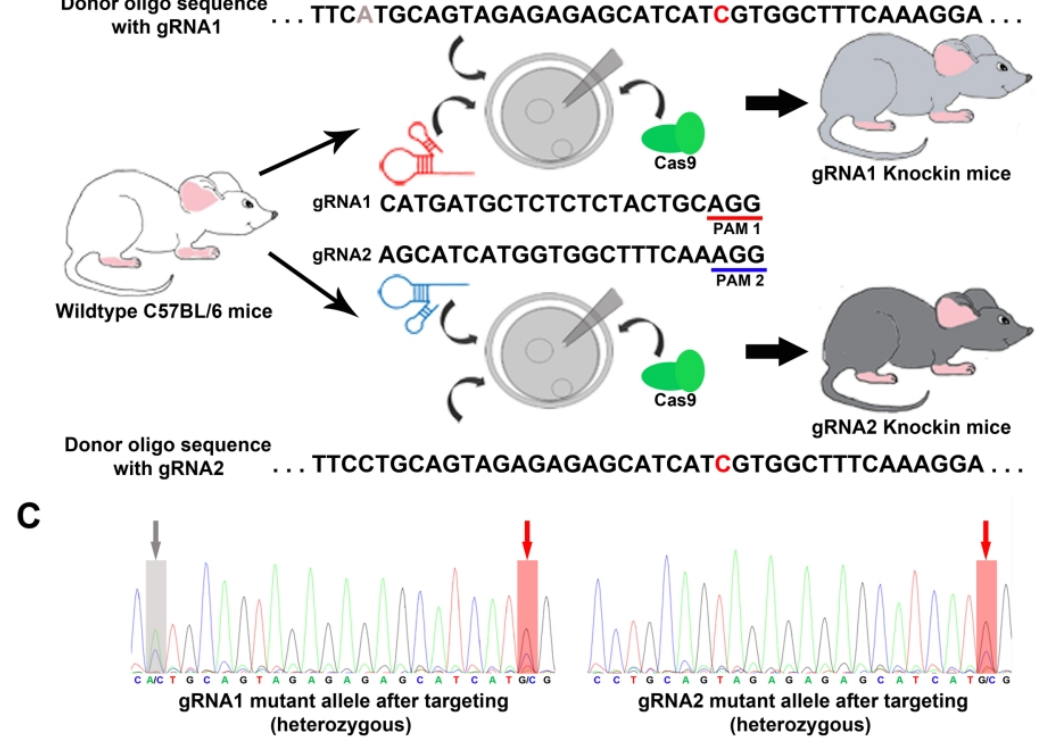

Figure 1

Figure 1. Generation of OAP-null mouse using the CRISPR/Cas9 system. A) Schematic representation of the targeted exon 1 of mouse AQP4 on chromosome 18. Coding exons are shown as solid black bars and are interspaced by non-coding sequences represented with thin lines. Two overlapping gRNAs, gRNA1 (red line) and gRNA2 (blue line), were used to target different AQP4 sequences of the wildtype allele. The proto spacer-adjacent motif (PAM) sequences are underlined for each gRNA (PAM1 for gRNA1 and PAM2 for gRNA2) while the single base (G) to be mutated is highlighted by an asterisk in the wildtype allele before targeting. The green scissors indicate the Cas9 cleavage site. The mutated base " $C$ " $(G \Rightarrow C)$ is highlighted in red in the mutant alleles obtained after targeting. The mutated allele obtained with gRNA1 displayed an additional silent mutation (in grey) at the PAM sequence. B) The genome of wildtype mice with C57BL/6 genetic background was edited by injecting Cas9 (in green), donor oligo sequences containing desired mutations and gRNAs (gRNA1 in red and gRNA2 in blue) in fertilized eggs. C) Sequence analysis of the

mutant alleles in heterozygous mice. The desired mutation $\mathrm{G} \Rightarrow C$ (red arrows) in the AQP4 gene was confirmed in both gRNA1 and gRNA2 strains of KI mice. The silent mutation $(C \Rightarrow A)$ at the PAM sequence is shown in grey for gRNA1 mutant allele. 
John Wiley \& Sons, Inc. 
A

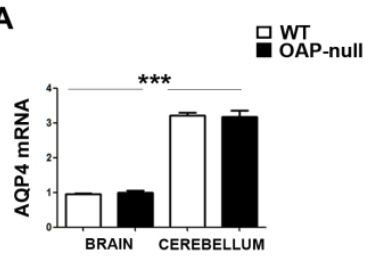

B

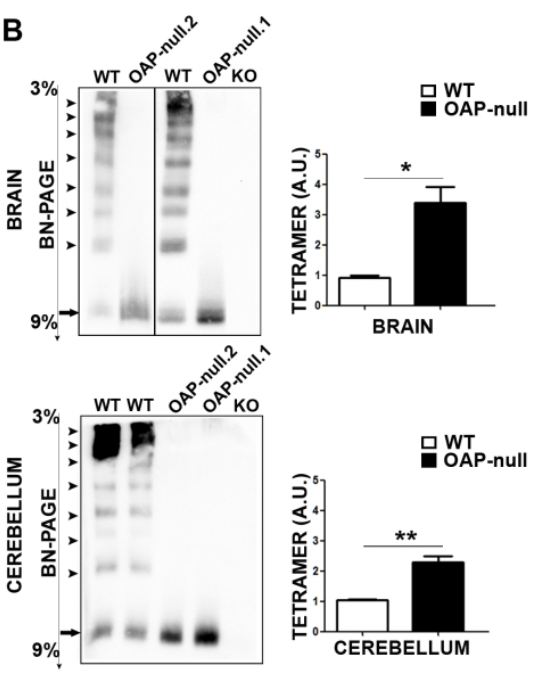

C

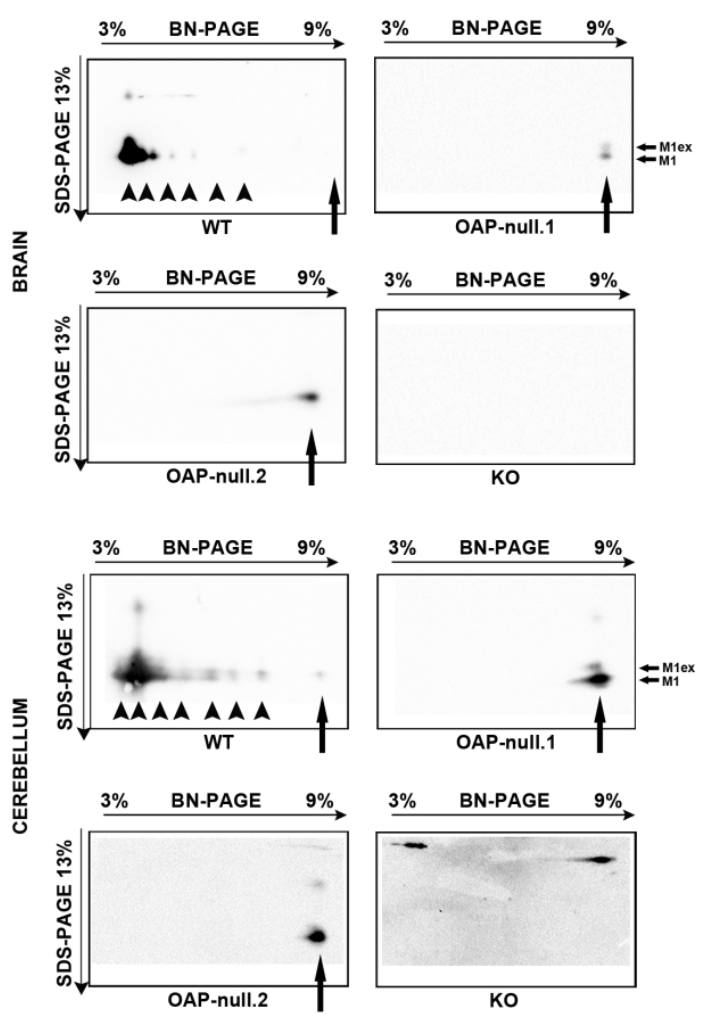

Figure 2

Figure 2. Analysis of AQP4 mRNA by qPCR and AQP4 assemblies by BN-PAGE and BN/SDS-PAGE in WT and OAP-null brain and cerebellum. A) Real time PCR analysis of relative AQP4 mRNA levels in the brain and cerebellum from WT and OAP-null mice. AQP4 mRNA levels were significantly higher in the cerebellum compared with the brain but there were no significant differences between tissues from WT and OAP-null mice (unpaired Student's t-test: ${ }^{* * *} \mathrm{P}<0.001, \mathrm{n}=3$. B) Left: membrane proteins extracted from the brain and cerebellum of OAP-null.1, OAP-null.2 and WT mice were separated under native conditions on 3-9\% acrylamide gels (BN-PAGE) and then subjected to immunoblot using AQP4 antibodies against all AQP4 isoforms (AQP4 Ab). Tissues from AQP4 KO mice (KO) were used as negative controls. In WT tissues, AQP4 tetramers (arrow) and several different pools corresponding to different sized AQP4 assemblies (arrowheads) are visualized. In contrast, in OAP-null tissues only the smaller AQP4 pool corresponding to AQP4 tetramers is visualized. Right: Bar graph summarizing the densitometric analysis, shown as arbitrary units (A.U.), performed on AQP4 tetramers indicated by the arrow in the tissues, as indicated. Data from OAP null.1 and OAP-null.2 have been pooled together since no significant difference was found between the two strains (unpaired Student's t-test: $* P<0.05, * * P<0.01, n=3-6$ ). Data in A) and B) are expressed as means \pm standard error of the mean (SEM). C) Two-dimensional analysis of AQP4 assemblies in WT and OAP-null brain and cerebellum. After being separated under native conditions in 3-9\% acrylamide gels (BN- 
PAGE, first dimension), membrane proteins were subjected to a second dimension performed under denaturing condition (SDS-PAGE), using a $13 \%$ acrylamide gel. Immunoblot was performed using AQP4 Ab. Tissues from AQP4 KO (KO) mice were used as negative controls. AQP4 tetramers are indicated by vertical arrows and AQP4 assemblies by arrowheads. M1-AQP4 (M1) and M1-AQP4ex (M1ex) are indicated by horizontal arrows. 
A
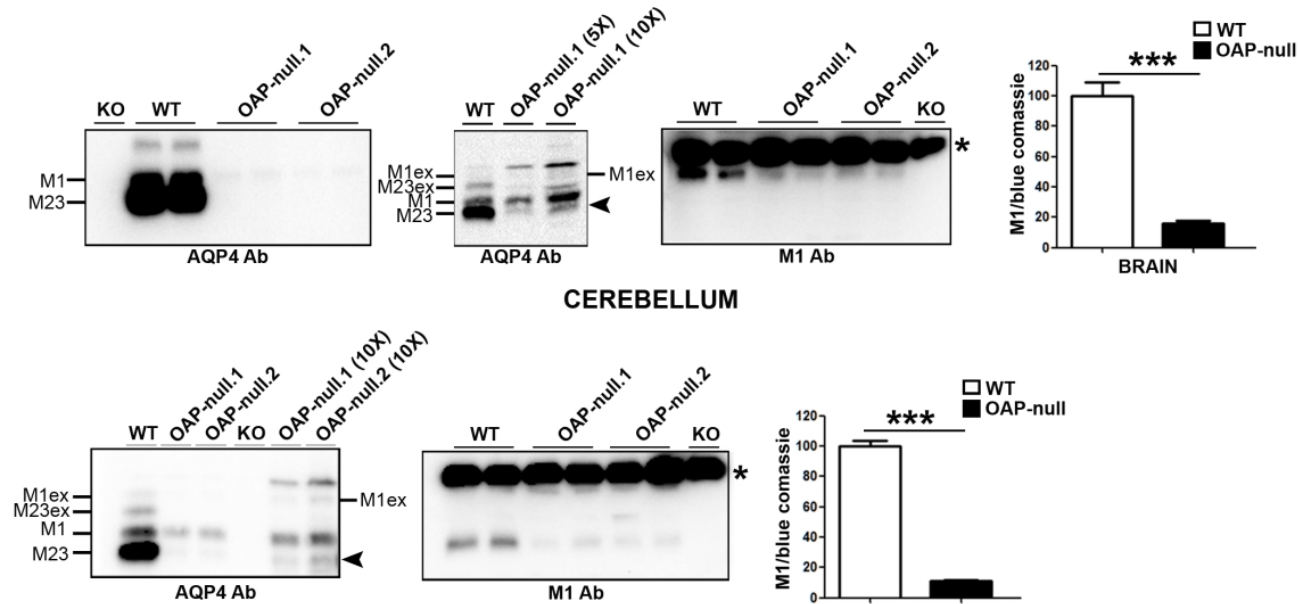

B

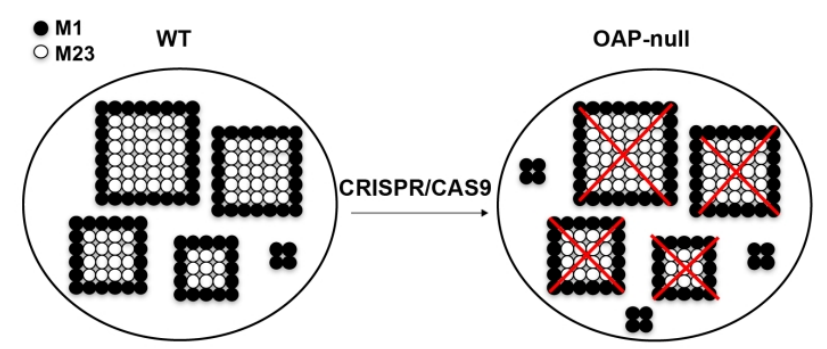

Figure 3

Figure 3. Western blot analysis of M1-AQP4 and M23-AQP4 expression in WT and OAP-null brain and cerebellum. A) Left: Western blot analysis of M1-AQP4 (M1) and M23-AQP4 (M23) expression in the brain and cerebellum from WT, OAP-null (OAP-null.1 and OAP-null.2) and AQP4 KO (KO) mice, performed using AQP4 $A b$ and antibodies able to recognize the M1-AQP4 isoform (M1 Ab) selectively. The asterisk indicates a non specific band recognized by $M 1 A b$ in all the brain and cerebellum tissues analyzed, including those from AQP4 KO mice, differing in size between brain and cerebellum. In the Western blot performed with AQP4 Ab, a ten-times $(10 X)$ or a 5 times (5X) higher concentration of OAP null tissues was loaded to visualize the correct expression of the M1-AQP4 isoform. In the $5 \mathrm{X}$ and 10X samples from OAP-null mice, the M1-AQP4ex

isoform (M1ex) and a band presumably corresponding to AQP4f (arrowhead) are indicated. Right: bar graphs summarizing the densitometric analysis of M1-AQP4 (M1/ Coomassie Blue, shown in \%) quantified from Western blot performed using M1 Ab in brain and cerebellum, as indicated. Coomassie Blue staining of total proteins was used as an internal control for protein loading (unpaired Student's t-test: $* * * P<0.001$, $\mathrm{n}=6$ ). Data are expressed as means \pm SEM. B) Schema showing the effect of M23-AQP4 deletion on its molecular partner M1-AQP4 and on AQP4 tetramer expression based on the data shown in Figure 2B and Figure 3A. 
A

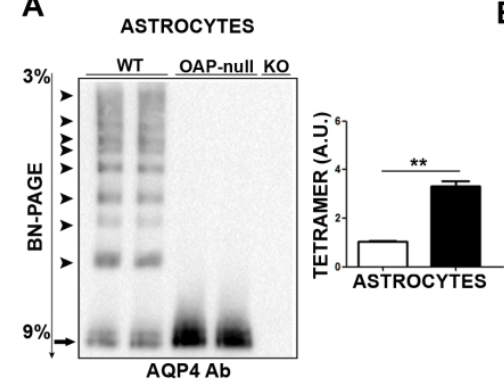

C

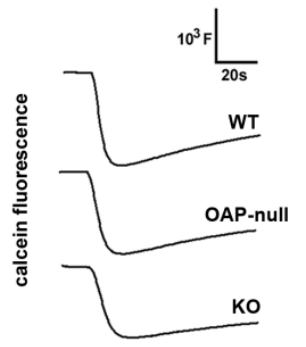

B

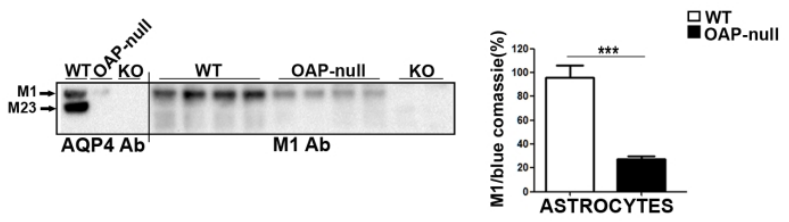

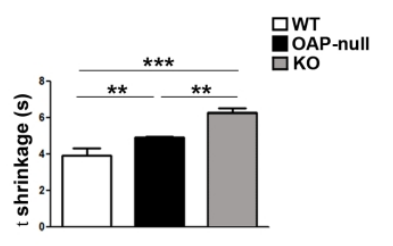

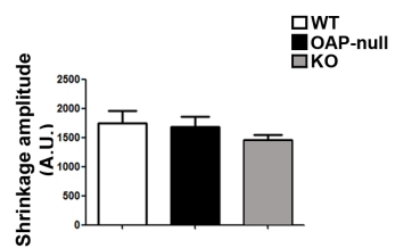

Figure 4

Figure 4. Analysis of AQP4 assemblies in WT and OAP-null astrocyte primary cultures by BN-PAGE, Western blot, and water transport assay. A) Left: Membrane proteins extracted from OAP-null and WT astrocytes were separated under native conditions in 3-9\% acrylamide gels (BN-PAGE) and then subjected to immunoblot analysis using AQP4 Ab. Astrocytes from AQP4 KO mice (KO) were used as negative controls. AQP4 tetramers are indicated by an arrow and AQP4 assemblies by arrowheads. Right: bar graph summarizing the densitometric analysis, shown as arbitrary units (A.U.) performed on AQP4 tetramer in WT and OAP-null astrocytes (unpaired Student's t-test: $* * P<0.01, n=3$ ). B) Left: Western blot analysis of AQP4 expression in astrocytes from WT, OAP-null and AQP4 KO (KO) mice, as indicated, performed using AQP4 Ab and M1 Ab. Right: bar graph summarizing the densitometric analysis of M1-AQP4 (M1/ Coomassie Blue, shown in \%) quantified from Western blot performed using M1 Ab in WT and OAP-null astrocyte primary cultures. Coomassie Blue staining was used as an internal control for protein loading (unpaired Student's t-test: $* * * P<0.001, n=3)$. C) Left: Functional analysis for AQP4 mediated water transport performed with the calcein quenching assay using the Flex Station multiplate reader. The typical shrinkage kinetics induced by osmotic shocks (hypertonic solution) and recorded from calcein-AM loaded WT, OAP-null and AQP4 KO (KO) astrocytes is shown. Middle: Bar graph of the mean \pm SEM values of time constants obtained by fitting an exponential curve to the shrinkage of cells after exposure to $360 \mathrm{mOsm}$ solution. Right: Bar graph showing the mean \pm SEM values of the maximal amplitude of astrocyte shrinkage (ANOVA followed by Newman-Keuls test: $* \mathrm{P}<0.05, * * \mathrm{P}<0.01 ; * * * \mathrm{P}<0.001, \mathrm{n}=3$ ). 
A
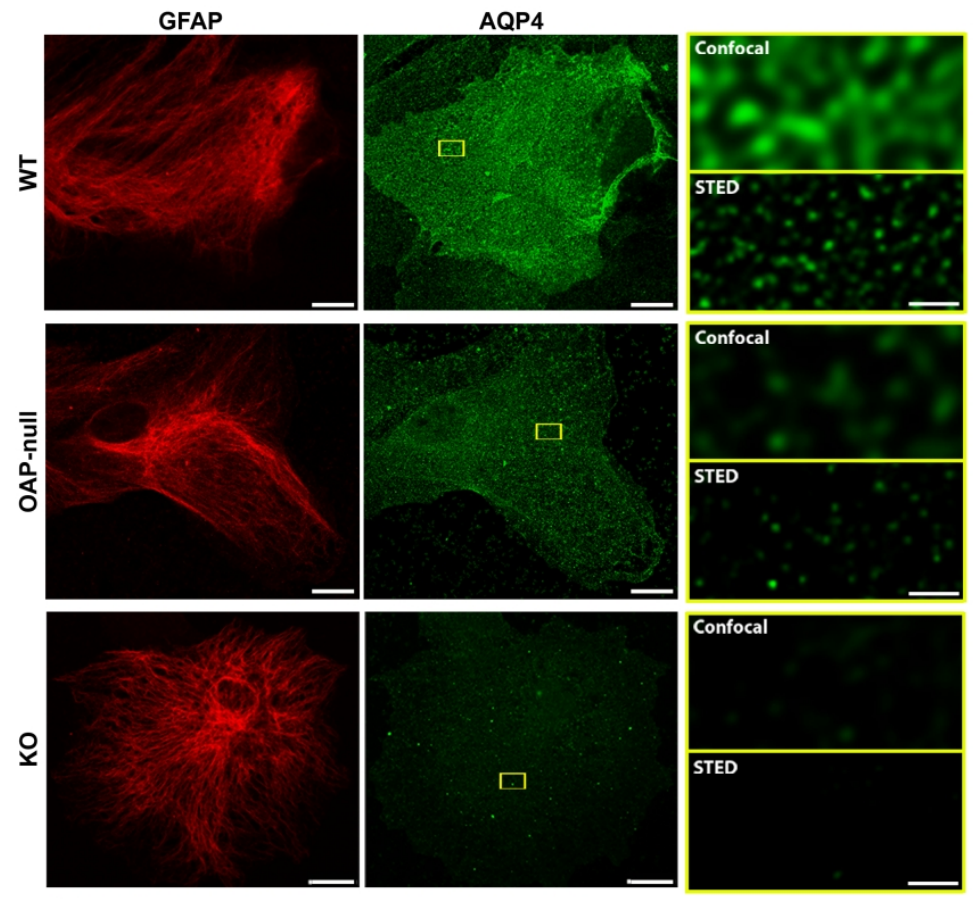

B
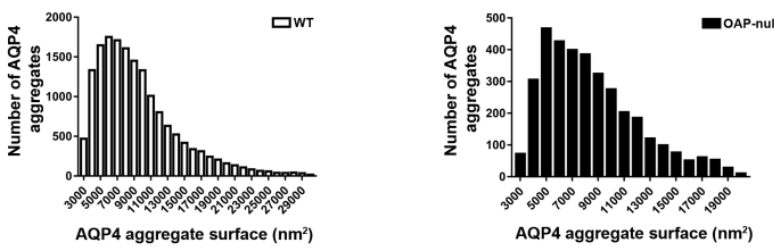

C

D
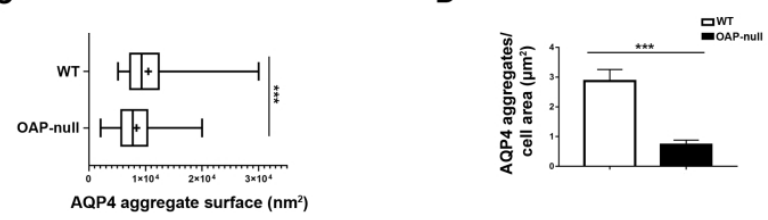

Figure 5

Figure 5. Analysis of AQP4 assemblies in WT and OAP-null astrocyte primary cultures by gSTED microscopy. A) Left and middle panels: representative images of WT, OAP-null and KO astrocytes, stained with AQP4 Ab (green) and GFAP Ab (red) obtained by confocal microscopy. Right panels: boxed yellow areas are shown at higher magnification with AQP4-aggregates imaged by confocal (upper insets) and gSTED (lower insets) microscopy, as indicated. Scale bars: $10 \mu \mathrm{m}$ (whole-cell); $500 \mathrm{~nm}$ (confocal and STED insets). B) AQP4aggregate surface ( $\mathrm{nm} 2$ ) frequency histogram of WT (left) and OAP-null (right) astrocytes, showing the number of analyzed clusters plotted against the cluster dimension. C) Differences in AQP4-aggregate median size between genotypes. In the box plot, the box represents the interquartile range (IQR), the central line is the median, the plus sign ' + ' identifies the mean values, and the whiskers extend from the minimum to the maximum values (Shapiro-Wilk normality test $* * * \mathrm{P}<0.001$ followed by unpaired Mann-Whitney test: $* * * \mathrm{P}$ $<0.001, n=3$ ). D) Quantification of AQP4-aggregate density expressed as the number of STED-identified clusters normalized to the cell surface area. Data are presented as mean \pm SEM (unpaired Student T-test: $* * * \mathrm{P}<0.001, \mathrm{n}=3)$. 
John Wiley \& Sons, Inc. 


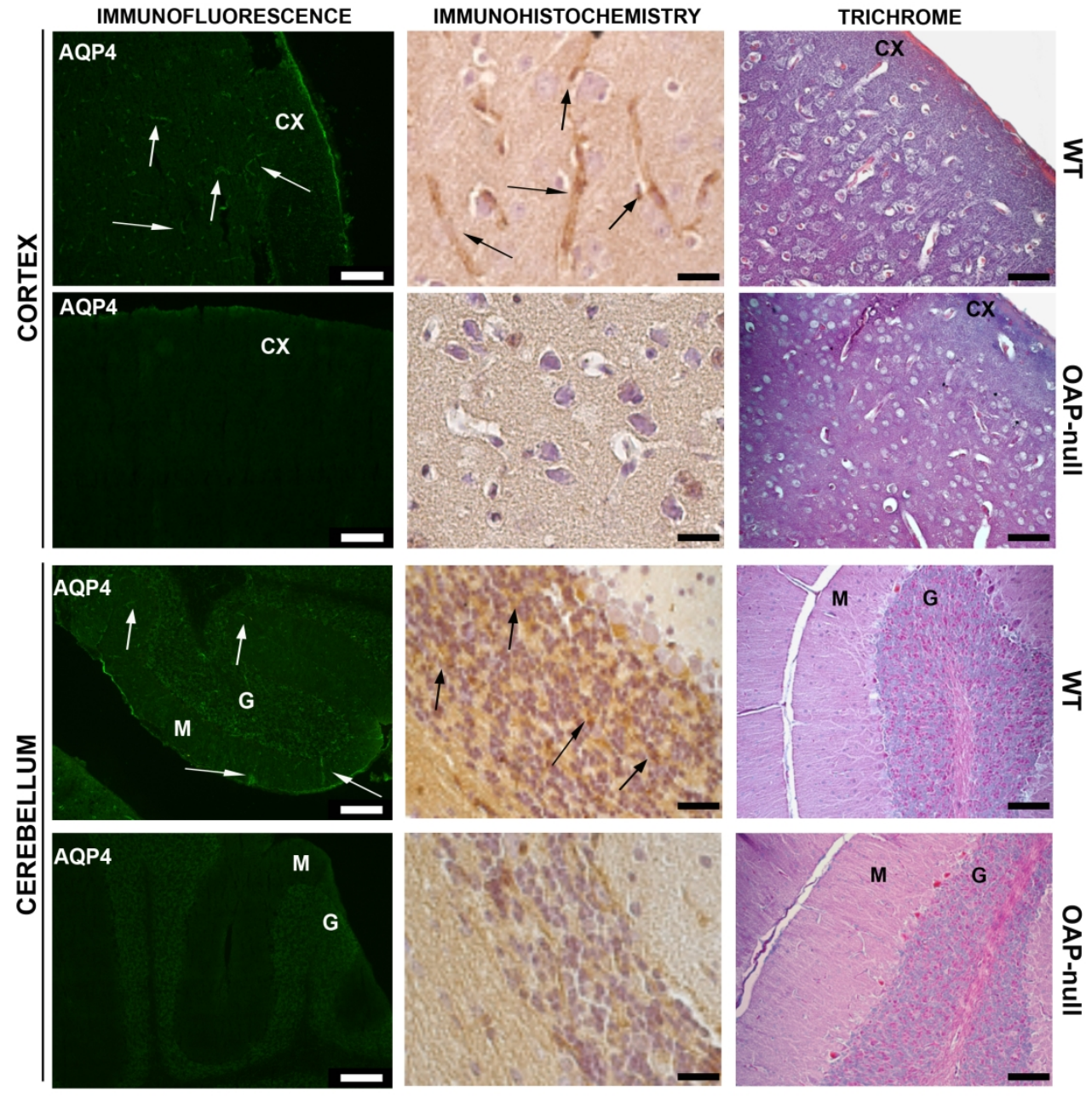

Figure 6

Figure 6. AQP4 distribution and morphological analysis on OAP-null and WT brain and cerebellum. AQP4 localization by immunofluorescence, immunoperoxidase and trichrome stain of brain cortex and cerebellum sections prepared from WT and OAP-null mice. Arrows indicate AQP4 staining which is clearly visible and specific in the WT tissues and very faint and diffuse in OAP-null tissues. CX, cortex; M, molecular cell layer; G, granular cell layer. Scale bar: $100 \mu \mathrm{m}$. 


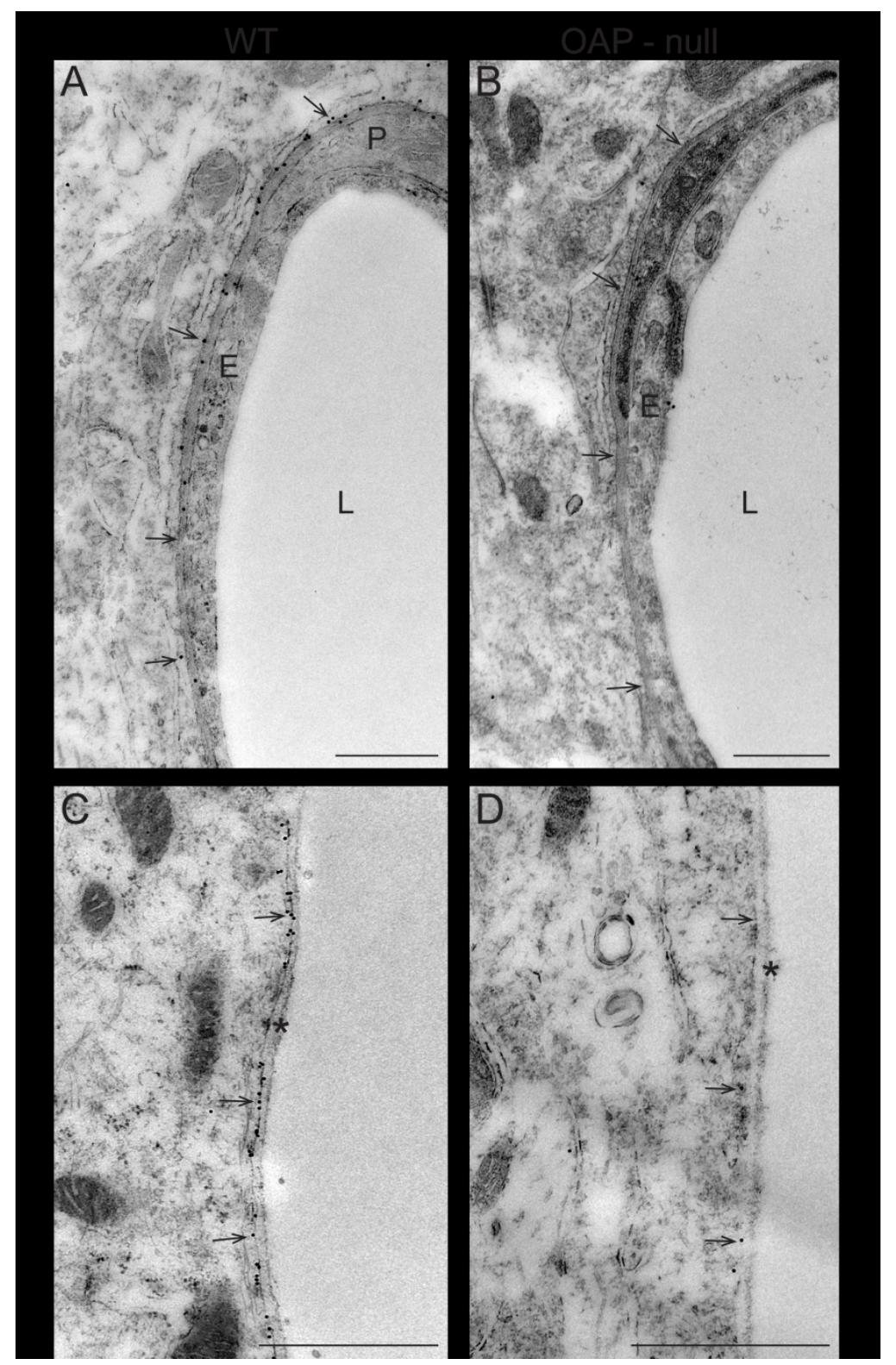

Figure 7. AQP4 immunogold labeling in OAP-null and WT cortex. Electron micrographs showing AQP4 immunogold labeling in cortex of WT ( $A$ and $C$ ) and OAP-null ( $B$ and D) mice. No AQP4 immnunogold labeling is visible at the perivascular astrocytic (B) membrane and only a few immunogold particles are present at the subpial endfoot (D) of the OAP-null mouse cortex. A and B are from perivascular membranes (arrows), while $C$ and $D$ are from subpial membranes (arrows). $L=$ lumen, $E=$ endothelium, $P=$ pericyte, asterisk = basal membrane. Scale bars (A, B: $500 \mathrm{~nm} ;$ C, D: $1 \mu \mathrm{m})$. 


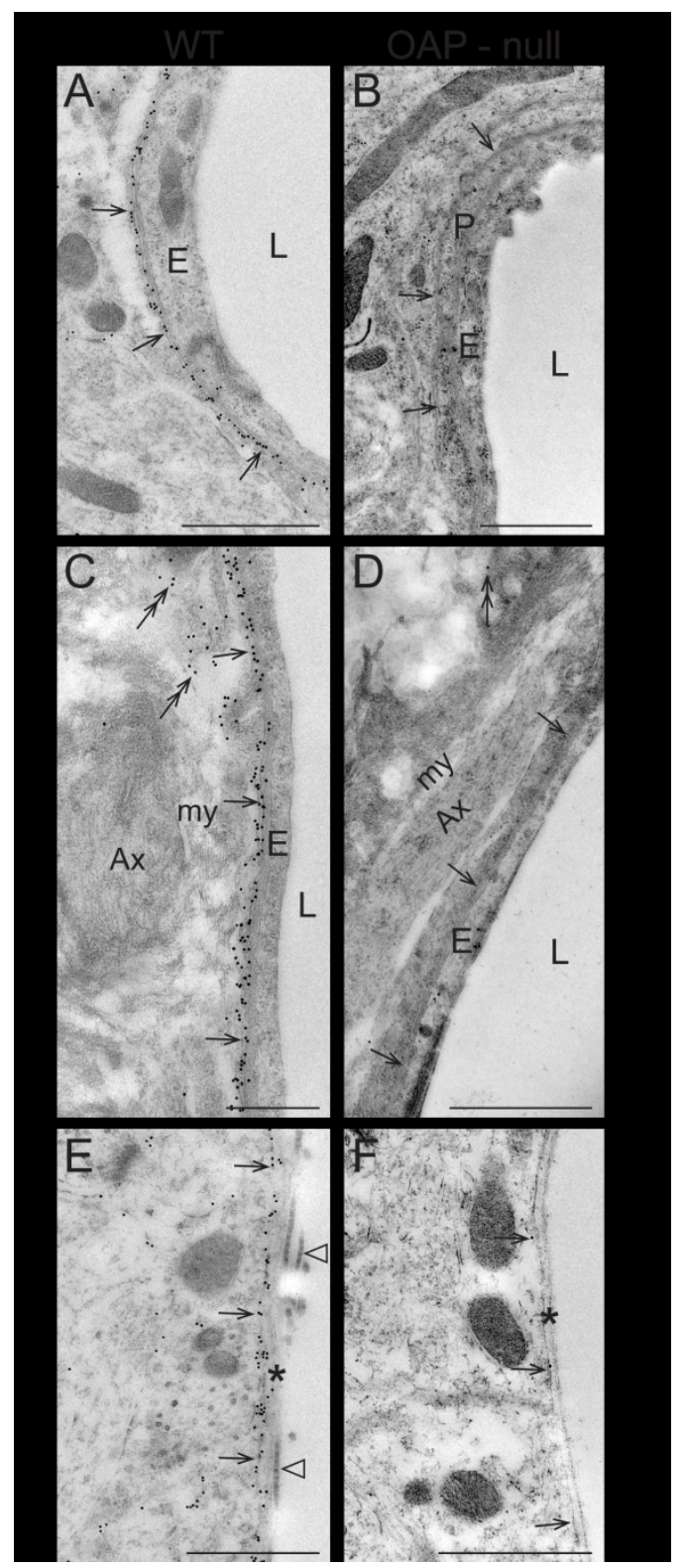

Figure 8. AQP4 immunogold labeling o in OAP-null and WT cerebellum. Electron micrographs showing AQP4 immunogold labeling in cerebellum of WT $(A, C, E)$ and OAP-null mice $(B, D, F)$. Note the strong reduction in the perivascular and subpial AQP4 immunogold labeling of the OAP-null mice compared to the WT. Immunogold particles indicating AQP4 labeling in astrocyte processes in the white matter of cerebellum are shown with double-headed arrows. A and B are from perivascular membranes (arrows) in the granule layer.

$C$ and $D$ are from perivascular membranes (arrows) in the white matter. $E$ and $F$ are from subpial membranes (arrows). $\mathrm{L}=$ lumen, $\mathrm{E}=$ endothelium, $\mathrm{P}=$ pericyte, $\mathrm{Ax}=$ axon, $\mathrm{my}=$ myelin, doubled arrows

$=$ other astrocyte membranes, asterisk = basal membrane, arrowheads = collagen fibers of pial mater. Scale bars (C: $500 \mathrm{~nm}$; A, B, D-F: $1 \mu \mathrm{m}$ ). 


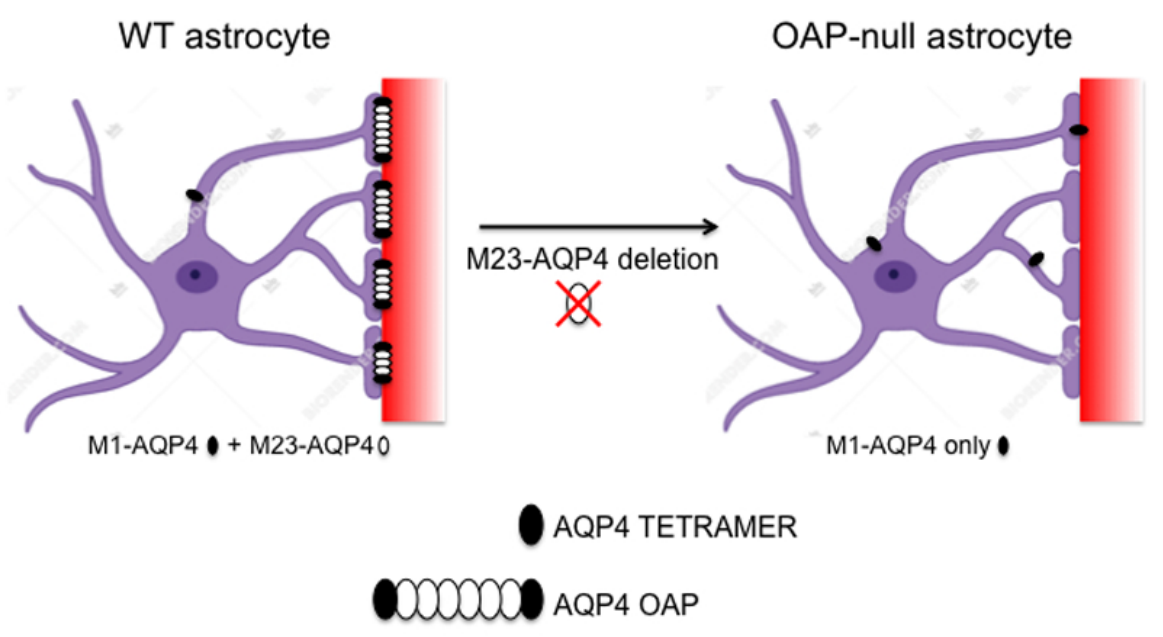

Graphical Abstract

$57 \times 42 \mathrm{~mm}(400 \times 400$ DPI $)$ 
A
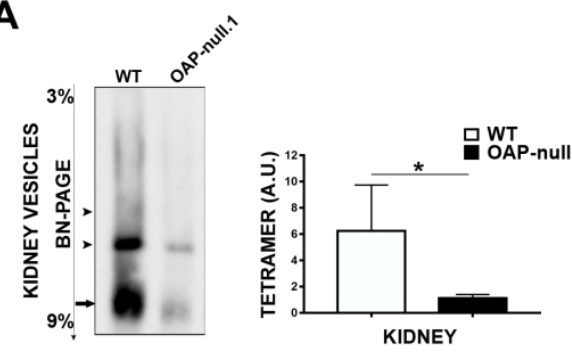

C

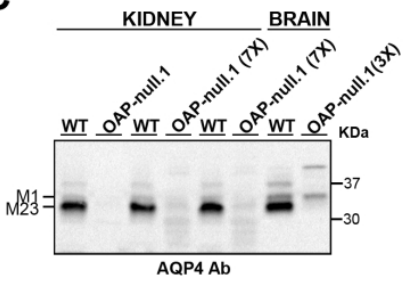

B

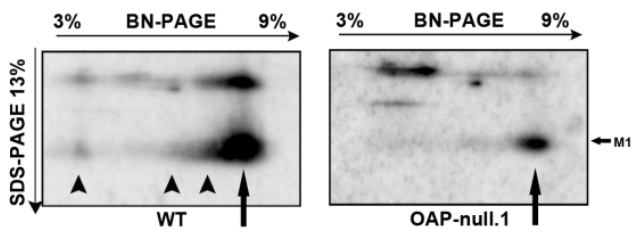

Figure 4 Supplementary Information.

Figure 4 Supplementary Information. Analysis of AQP4 assemblies and M1-AQP4 and M23-AQP4 expression by BN-PAGE, BN/SDS-PAGE and Western blot in WT and OAP-null kidney plasma membrane vesicles. Membrane proteins were extracted from plasma membrane vesicles prepared from the kidney medulla of OAP-null and WT mice as follows: mouse tissue was cut into small pieces and homogenized with a Dounce apparatus in five volumes of ice-cold Homogenizing Buffer $(250 \mathrm{mM}$ Sucrose; $10 \mathrm{mM} \mathrm{Tris-} \mathrm{HCl}, \mathrm{pH} \mathrm{7,5})$ added with protease inhibitor cocktail. Homogenates were centrifuged at $4,000 \mathrm{xg}$ for 10 minutes at $4^{\circ} \mathrm{C}$. After centrifugation, pellets were discarded and supernatants collected and centrifuged at $17,000 \times \mathrm{xg}$ for 45 minutes at $4^{\circ} \mathrm{C}$ to obtain a low-speed membrane vesicles-enriched pellet. The resulting pellets were resuspended in seven volumes of BN-PAGE lysis buffer added with protease inhibitor for one hour on ice and vortexed every 10 minutes. Vesicle lysates were then centrifuged at $21,000 \mathrm{xg}$ for one hour at $4^{\circ} \mathrm{C}$. The supernatant was collected and the protein content was measured using the BCATM Protein Assay Kit

(http://www.thermoscientific.com).

A) Extracted proteins were separated under native conditions on 3-9\% acrylamide gels (BN-PAGE) and then subjected to immunoblot using AQP4 antibodies against all AQP4 isoforms (AQP4 Ab). AQP4 tetramers are indicated by the arrow and pools corresponding to AQP4 assemblies are indicated by arrowheads. Right: Bar graph summarizing the densitometric analysis, shown as arbitrary units (A.U.), performed on AQP4

tetramers indicated by the arrow in the tissues, as indicated (unpaired Student's t-test, $* \mathrm{P}<0.05, \mathrm{n}=3$ ). Data are expressed as means \pm standard error of the mean (SEM). B) Two-dimensional analysis of AQP4 assemblies in WT and OAP-null kidney plasma membrane vesicles. After being separated under native conditions in 3-9\% acrylamide gels (BN-PAGE, first dimension), membrane proteins were subjected to a second dimension performed under denaturing condition (SDS-PAGE), using a $13 \%$ acrylamide gel. Immunoblot was performed using AQP4 Ab. AQP4 tetramers are indicated by vertical arrows and AQP4 assemblies by arrowheads. C) Western blot analysis of M1-AQP4 (M1) and M23-AQP4 (M23) expression in 
the vesicles from WT and OAP-null mice, performed using AQP4 Ab. Brain tissues were used as control. A seven-times $(7 X)$ or three-times $(3 X)$ higher concentration of OAP null tissues was loaded to visualize the expression of the M1-AQP4 isoform.

The results show that no tetramer upregulation occurs in OAP-null kidneys and that M1-AQP4 expression is massively reduced and undetectable by Western blot even using a seven-times higher concentration of protein sample compared with the WT. 